This item was submitted to Loughborough's Research Repository by the author.

Items in Figshare are protected by copyright, with all rights reserved, unless otherwise indicated.

\title{
Synchronization of generally uncertain Markovian inertial neural networks with random connection weight strengths and image encryption application
}

\section{PLEASE CITE THE PUBLISHED VERSION}

https://doi.org/10.1109/tnnls.2021.3131512

\section{PUBLISHER}

Institute of Electrical and Electronics Engineers (IEEE)

VERSION

AM (Accepted Manuscript)

\section{PUBLISHER STATEMENT}

(c) 2021 IEEE. Personal use of this material is permitted. Permission from IEEE must be obtained for all other uses, in any current or future media, including reprinting/republishing this material for advertising or promotional purposes, creating new collective works, for resale or redistribution to servers or lists, or reuse of any copyrighted component of this work in other works.

\section{LICENCE}

\section{All Rights Reserved}

\section{REPOSITORY RECORD}

Wang, Junyi, Zewen Ji, Huaguang Zhang, Zhanshan Wang, and Qinggang Meng. 2021. "Synchronization of Generally Uncertain Markovian Inertial Neural Networks with Random Connection Weight Strengths and Image Encryption Application". Loughborough University. https://hdl.handle.net/2134/17230748.v1. 


\title{
Synchronization of Generally Uncertain Markovian Inertial Neural Networks with Random Connection Weight Strengths and Image Encryption Application
}

\author{
Junyi Wang, Member, IEEE, Zewen Ji, Huaguang Zhang, Fellow, IEEE, Zhanshan Wang, Senior Member, IEEE, \\ Qinggang Meng, Senior Member, IEEE
}

\begin{abstract}
This paper focuses on the synchronization problem of delayed inertial neural networks (INNs) with generally uncertain Markovian jumping and their applications in image encryption. The random connection weight strengths and generally uncertain Markovian are discussed in INNs model. Compared with most existing INNs models that have constant connection weight strengths, our model is more practical because connection weight strengths of INNs may randomly vary owing to the external and internal environment and human factor. The delay-range-dependent synchronization conditions (DRDSC) could be obtained by adopting the delay-product-term LyapunovKrasovskii functional (DPTLKF) and higher order polynomial based relaxed inequality (HOPRII). In addition, the desired controllers are obtained by solving a set of linear matrix inequalities. Finally, two examples are shown to demonstrate the effectiveness of the proposed results.
\end{abstract}

Index Terms-Inertial neural networks, random connection weight strengths, delay-product-term Lyapunov-Krasovskii functional, higher order polynomial based relaxed inequality

\section{INTRODUCTION}

$\mathbf{I}$ $\mathrm{N}$ recent years, neural networks have received considerable attention because of their broad applications in pattern recognition, associative memories, signal processing and secure communication, etc [1]-[8]. Due to the finite switching speed of the amplifier, time delays often occur between neurons and may result in complicated chaotic dynamics [3][12].

There are many papers devoting to Markovian jumping systems (MJSs) because many practical systems experience random changes in their parameters and structures [13]-[27]. In [28], the stability problem of discrete-time linear systems with random jumping parameters was investigated, and necessary and sufficient conditions of mean square stability were obtained. The global exponential stability problem was addressed for delayed recurrent neural networks with Markovian jumping parameters in [29]. For relaxing the traditional assumption in MJSs, the stability analysis and stabilization problems of

This work was supported by the National Natural Science Foundation of China (61903075, U20A20197), and Project of Liaoning Province Science and Technology Program (2019-KF-03-02), and the Fundamental Research Funds for the Central Universities (N2026003).

J. Wang and Z. Ji are with Faculty of Robot Science and Engineering, Northeastern University, Shenyang, Liaoning 110819, China. H. Zhang, and Z. Wang are with College of Information Science and Engineering, Northeastern University, Shenyang, Liaoning 110819, China. Q. Meng is with Department of Computer Science, Loughborough University, Loughborough LE11 3TU, UK. (email: wjyi168@126.com; 1901944@stu.neu.edu.cn; hgzhang@ieee.org; wangzhanshan@ise.neu.edu.cn; Q.Meng@lboro.ac.uk). discrete-time Markovian linear systems with partially known transition probabilities were investigated in [30]. After that, the stability and synchronization of discrete-time neural networks were investigated based on mixed mode-dependent time delays and Markovian jumping in [31]. By considering the partially known transition rates, the stability and synchronization problems of Markovian jumping neural networks with time-varying delays were investigated in [20]. Based on [20] and [30], the finite-time stochastically stability problem of Markovian jumping memristive neural networks with partly unknown transition rates was concerned by introducing the Markovian switching Lyapunov functional. The delay-dependent robust fault detection problem of MJSs with partly unknown transition rates was addressed in [32]. Different from partly unknown transition rates, the passivity problem of Markovian jumping neural networks with piecewise-constant transition rates was studied in [33]. By fully considering the property of transition rates and the characteristic of uncertain domains, the stability problems of Markovian jumping linear systems with uncertain transition rates were proposed [34]. Based on [34] and [35], Markovian nonlinearly coupled neural networks with generally uncertain transition rates were investigated in [27], and the corresponding local synchronization conditions were proposed.

Inertial neural networks (INNs), as a kind of special neural networks, were described by the second-order differential equation [36]. In recent years, the INNs have attracted much attention [37]-[42]. The second-order term is named inertial term in INNs and could produce more complex dynamic behavior than normal neural networks with firstorder term. Based on integral inequality method, the finitetime synchronization conditions of INNs were proposed in [37]. The fixed-time synchronization of inertial memristorbased neural networks was investigated, and four different feedback controllers were designed in [40]. Because of the chaotic characteristics of INNs, they were utilized in image encryption/decryption [43] and [44]. The synchronization of Markovian INNs was investigated in [43], and the obtained results were applied in image encryption. Based on [43], the synchronization conditions of delayed INNs with generally Markovian jumping were proposed in [45]. In [45], the uncertain and unknown elements in the transition rates matrix are solved based on Schur complement and matrix inequality Lemma, which increases the dimension and computational burden of INNs synchronization conditions. In addition, the 
connection weight strengths of INNs may randomly vary because of environmental and artificial factors. It is necessary to further consider the synchronization and image encryption problems of INNS with random connection weight strengths.

Motivated by the foregoing discussions, this paper explores the synchronization of generally uncertain Markovian INNs with random connection weight strengths and their applications in image encryption. Based on delay-product-term Lyapunov - Krasovskii functional (DPTLKF) and higher order polynomial-based relaxed inequality (HOPRII) containing more information of upper and low bounds of time delay and time delay derivative, the new synchronization conditions and desired controllers are proposed to ensure drive system and response system synchronized in mean square. The contributions of this paper are summarized as follows.

1) Compared with most existing INNs with constant connection weight strengths [37]-[39], the INNs in this paper is more practical because connection weight strengths of INNs may randomly vary owing to the influence of device performances and the uncertainties of external environment.

2) The DPTLKF is adopted in this paper to deal with the synchronization of generally uncertain Markovian INNS with random connection weight strengths, which makes the synchronization conditions include more information about time-varying delay and its derivative.

3) Different from the existing generally uncertain Markovian NNs [45]-[47], Schur complement and matrix inequality Lemma are not adopted in the process of handling generally uncertain Markovian, and only one set of relaxation variables are utilized to deal with generally uncertain transition rates in this paper, which reduces the dimension and computational complexity of synchronization conditions. By in-depth exploiting relationships among the generally uncertain transition rates, the new synchronization criteria are derived for the INNs.

The rest of this paper is arranged as follows. In Section II, some preliminaries and generally uncertain INNs are introduced. In Section III, the synchronization criteria and controllers of INNs are proposed. In Section IV, two examples are given to demonstrate the validity of the proposed results. Finally, conclusions are drawn in Section V.

\section{Problem Statement and Preliminaries}

\section{A. Notation}

Notation: Throughout the article, $\mathcal{R}^{n}$ and $\mathcal{R}^{n \times n}$ denote $n$-dimensional Euclidean space and $n \times n$ real matrices, respectively. $\|\cdot\|$ stands for Euclidean vector norm. $A^{T}$ denotes the transpose of matrix $A$. When $A>0, A$ means a symmetric positive definite matrix. $\operatorname{Sym}\{A\}=A+A^{T} . E_{n}$ denotes the $n$-dimensional identity matrix. $\left(\begin{array}{cc}A & B \\ * & C\end{array}\right)$ stands for $\left(\begin{array}{cc}A & B \\ B^{T} & C\end{array}\right) \cdot \mathcal{E}$ denotes the mathematical expectation. $e_{x}=\left[0_{n \times(x-1) n}, E_{n}, 0_{n \times(17-x) n}\right](x=1,2, \cdots, 17)$.

\section{B. Problem Formulation}

$\left\{\imath_{t}, t \geq 0\right\}$ is a right-continuous Markovian process on the probability space, which takes values in a finite state space $\mathcal{C}=\{1,2, \cdots, \mathcal{M}\}$ with generator $\Omega=\left(\lambda_{a b}\right),(a, b \in \mathcal{C})$ given by

$$
\operatorname{Pr}\left\{\imath_{t+\Delta t}=b \mid \imath_{t}=a\right\}=\left\{\begin{array}{c}
\lambda_{a b} \Delta t+o(\Delta t), a \neq b, \\
1+\lambda_{a a} \Delta t+o(\Delta t), a=b,
\end{array}\right.
$$

where $\Delta t>0$ and $\lim _{\Delta t \rightarrow 0}(o(\Delta t) / \Delta t)=0 . \lambda_{a b} \geq 0(a \neq b)$ is the transition rate from mode $a$ at time $t$ to mode $b$ at time $t+\Delta t$, and $\lambda_{a a}=-\sum_{b=1, b \neq a}^{\mathcal{M}} \lambda_{a b}$.

In this paper, transition rates of the jumping process are generally uncertain. For instance, transition rate matrix (TRM) $\Omega$ with $\mathcal{M}$ operation modes may be expressed as

$$
\Omega=\left(\begin{array}{ccccc}
\tilde{\lambda}_{11}+\Delta_{11} & ? & \tilde{\lambda}_{13}+\Delta_{13} & \cdots & \tilde{\lambda}_{1 \mathcal{M}}+\Delta_{1 \mathcal{M}} \\
? & ? & \tilde{\lambda}_{23}+\Delta_{23} & \cdots & ? \\
\vdots & \vdots & \vdots & \ddots & \vdots \\
\tilde{\lambda}_{\mathcal{M} 1}+\Delta_{\mathcal{M} 1} & ? & ? & \cdots & \tilde{\lambda}_{\mathcal{M M}}+\Delta_{\mathcal{M M}}
\end{array}\right)
$$

where "?" denotes the completely unknown transition rate. $\tilde{\lambda}_{a b}$ and $\triangle_{a b}$ denote the estimate value and estimate error of the uncertain transition rate $\lambda_{a b}$, respectively. $\left\|\triangle_{a b}\right\| \leq \varpi_{a b}$ and $\varpi_{a b} \geq 0 . \tilde{\lambda}_{a b}, \varpi_{a b}$ are known. For the clarity of the notation, $\forall a \in \mathcal{C}$, we denote $\mathcal{C}^{a}=\mathcal{C}_{k}^{a} \cup \mathcal{C}_{u k}^{a}$, where $\mathcal{C}_{k}^{a}=\left\{b:\right.$ The estimate value of $\lambda_{a b}$ is known for $\left.b \in \mathcal{C}\right\}$ and $\mathcal{C}_{u k}^{a}=\left\{b:\right.$ The estimate value of $\lambda_{a b}$ is unknown for $\left.b \in \mathcal{C}\right\}$. On the basis of the characteristics of the transition rates, the following cases are assumed.

If $\mathcal{C}_{k}^{a} \neq \mathcal{C}$, and $a \notin \mathcal{C}_{k}^{a}$, then $\tilde{\lambda}_{a b}-\varpi_{a b} \geq 0,\left(\forall b \in \mathcal{C}_{k}^{a}\right)$.

If $\mathcal{C}_{k}^{a} \neq \mathcal{C}$, and $a \in \mathcal{C}_{k}^{a}$, then $\tilde{\lambda}_{a b}-\varpi_{a b} \geq 0,\left(\forall b \in \mathcal{C}_{k}^{a}, b \neq a\right)$, $\tilde{\lambda}_{a a}+\varpi_{a a} \leq 0$, and $\sum_{b \in \mathcal{C}_{k}^{a}} \tilde{\lambda}_{a b} \leq 0$.

If $\mathcal{C}_{k}^{a}=\mathcal{C}$, then $\tilde{\lambda}_{a b}-\varpi_{a b} \geq 0,(\forall b \in \mathcal{C}, b \neq a), \tilde{\lambda}_{a a}=$ $-\sum_{b=1, b \neq a}^{\mathcal{M}} \tilde{\lambda}_{a b} \leq 0$, and $\varpi_{a a}=\sum_{b=1, b \neq a}^{\mathcal{M}} \varpi_{a b} \geq 0$.

Remark 1: Different from most existing TRM, the generally uncertain TRM (1) is considered for INNs in this paper, which is more general and applicable. When $\triangle_{a b}=0$, generally uncertain TRM (1) is reduced to partially unknown TRM. When $\mathcal{C}_{k}^{a}=\mathcal{C}(\forall b \in \mathcal{C})$, generally uncertain TRM (1) is reduced to bounded uncertain TRM.

Consider the INNs as follows

$$
\begin{aligned}
& \frac{d^{2} u_{k}(t)}{d t^{2}}=-a_{k}\left(\imath_{t}\right) \frac{d u_{k}(t)}{d t}-b_{k}\left(\imath_{t}\right) u_{k}(t)+\alpha(t) \sum_{l=1}^{n} w_{k l}^{1}\left(\imath_{t}\right) \\
& f_{l}\left(u_{l}(t)\right)+\beta(t) \sum_{l=1}^{n} w_{k l}^{2}\left(\imath_{t}\right) f_{l}\left(u_{l}(t-\tau(t))\right)+T_{k}
\end{aligned}
$$

where $k=1,2, \cdots, n, u_{k}(t)$ denotes the state of the $k t h$ neuron at time $t . a_{k}\left(\imath_{t}\right)>0, b_{k}\left(\imath_{t}\right)>0$ are constants. $w_{k l}^{1}\left(l_{t}\right)$ and $w_{k l}^{2}\left(\imath_{t}\right)$ are connection weights related to the neurons. $f_{l}($. is the activation function of $l$ th neurons with $f_{l}(0)=0 . T_{k}$ is the external input of the $k t h$ neuron at time $t$. The time-varying delay $\tau(t)$ satisfies $0 \leq \tau(t) \leq \tau, \mu_{1} \leq \dot{\tau}(t) \leq \mu_{2}$, where $\tau, \mu_{1}$ and $\mu_{2}$ are constants. $\alpha(t), \beta(t)$ denote the random 
connection weight strengths. The initial condition associated with (2) is given as follows: $u_{k}(s)=\phi_{k}(s), \frac{d u_{k}(s)}{d s}=\tilde{\phi}_{k}(s)$, $s \in([-\tau, 0])$ and $\phi_{k}(t), \tilde{\phi}_{k}(t) \in \mathcal{C}([-\tau, 0], \mathcal{R})$. The mathematical expectations and variances of $\alpha(t)$ and $\beta(t)$ are $\mathcal{E}\{\alpha(t)\}=\bar{\alpha}, \mathcal{E}\left\{(\alpha(t)-\bar{\alpha})^{2}\right\}=\nu_{\alpha}, \mathcal{E}\{\beta(t)\}=\bar{\beta}$ and $\mathcal{E}\left\{(\beta(t)-\bar{\beta})^{2}\right\}=\nu_{\beta}$.

Remark 2: Different from most existing INNs [37], [42][45] with constant connection weight strengths, the random connection weight strengths are considered for INNS in this paper, which is more practical because connection weight strengths of INNs may randomly vary owing to environmental and artificial interferences.

Assumption 1 [48] For any $u_{1}, u_{2} \in \mathcal{R}$, there are constants $l_{k}^{-}, l_{k}^{+}$, such that

$$
l_{k}^{-} \leq \frac{f_{k}\left(u_{1}\right)-f_{k}\left(u_{2}\right)}{u_{1}-u_{2}} \leq l_{k}^{+}, k=1,2, \cdots, n .
$$

We denote

$$
L_{1}=\operatorname{diag}\left\{l_{1}^{+} l_{1}^{-}, \cdots, l_{n}^{+} l_{n}^{-}\right\}, L_{2}=\operatorname{diag}\left\{\frac{l_{1}^{+}+l_{1}^{-}}{2}, \cdots, \frac{l_{n}^{+}+l_{n}^{-}}{2}\right.
$$

For constant $\mho_{k}$, the following transformation is employed

$$
b_{k}(t)=\frac{d u_{k}(t)}{d t}+\mho_{k} u_{k}(t), k=1,2, \cdots, n .
$$

Then, system (2) is rewritten as the following form

$$
\begin{aligned}
\frac{d u_{k}(t)}{d t}= & -\mho_{k} u_{k}(t)+b_{k}(t) \\
\frac{d b_{k}(t)}{d t}= & -\left[b_{k}\left(\imath_{t}\right)+\mho_{k}\left(\mho_{k}-a_{k}\left(\imath_{t}\right)\right)\right] u_{k}(t)-\left(a_{k}\left(\imath_{t}\right)-\mho_{k}\right) \\
& \times b_{k}(t)+\alpha(t) \sum_{l=1}^{n} w_{k l}^{1}\left(\imath_{t}\right) f_{l}\left(u_{l}(t)\right) \\
& +\beta(t) \sum_{l=1}^{n} w_{k l}^{2}\left(\imath_{t}\right) f_{l}\left(u_{l}(t-\tau(t))\right)+T_{k} \\
= & -\tilde{a}_{k}\left(\imath_{t}\right) u_{k}(t)-\tilde{b}_{k}\left(\imath_{t}\right) b_{k}(t)+\alpha(t) \sum_{l=1}^{n} w_{k l}^{1}\left(\imath_{t}\right) \\
& \times f_{l}\left(u_{l}(t)\right)+\beta(t) \sum_{l=1}^{n} w_{k l}^{2}\left(\imath_{t}\right) f_{l}\left(u_{l}(t-\tau(t))\right)+T_{k},
\end{aligned}
$$

where $\tilde{a}_{k}\left(\imath_{t}\right)=b_{k}\left(\imath_{t}\right)+\mho_{k}\left(\mho_{k}-a_{k}\left(\imath_{t}\right)\right), \tilde{b}_{k}\left(\imath_{t}\right)=a_{k}\left(\imath_{t}\right)-$ $\mho_{k}$. The initial condition associated with (4) is considered as follows $u_{k}(s)=\phi_{k}(s), \frac{d u_{k}(s)}{d s}=\tilde{\phi}_{k}(s), b_{k}(s)=\tilde{\phi}_{k}(s)+$ $\mho_{k} \phi_{k}(s)$, and $s \in([-\tau, 0])$.

Now, system (4) is rewritten as the following form

$$
\begin{aligned}
\frac{d u(t)}{d t}= & -A u(t)+b(t), \\
\frac{d b(t)}{d t}= & -B\left(\imath_{t}\right) b(t)-C\left(\imath_{t}\right) u(t)+\alpha(t) W^{1}\left(\imath_{t}\right) f(u(t)) \\
& +\beta(t) W^{2}\left(\imath_{t}\right) f(u(t-\tau(t)))+T,
\end{aligned}
$$

where $u(t)=\left[u_{1}(t), u_{2}(t), \ldots, u_{n}(t)\right]^{T}, \quad b(t)=$ $\left[b_{1}(t), b_{2}(t), \ldots, b_{n}(t)\right]^{T}, \quad A=\operatorname{diag}\left\{\mho_{1}, \cdots, \mho_{n}\right\}$, $B\left(\imath_{t}\right)=\operatorname{diag}\left\{a_{1}\left(\imath_{t}\right)-\mho_{1}, \cdots, a_{n}\left(\imath_{t}\right)-\mho_{n}\right\}, C\left(\imath_{t}\right)=$ $\operatorname{diag}\left\{b_{1}\left(\imath_{t}\right)+\mho_{1}\left(\mho_{1}-a_{1}\left(\imath_{t}\right)\right), \cdots, b_{n}\left(\imath_{t}\right)+\mho_{n}\left(\mho_{n}-a_{n}\left(\imath_{t}\right)\right)\right\}$,
$W^{1}\left(\imath_{t}\right)=\left(w_{k l}^{1}\left(\imath_{t}\right)\right)_{n \times n}, \quad W^{2}\left(\imath_{t}\right)=\left(w_{k l}^{2}\left(\imath_{t}\right)\right)_{n \times n}$, $T=\left[T_{1}, \cdots, T_{n}\right]$.

To be more convenient, each possible value of $\imath_{t}$ is denoted by $a(a \in \mathcal{C})$. According to (5), one gets

$$
\begin{aligned}
\frac{d u(t)}{d t}= & -A u(t)+b(t), \\
\frac{d b(t)}{d t}= & -B_{a} b(t)-C_{a} u(t)+\alpha(t) W_{a}^{1} f(u(t)) \\
& +\beta(t) W_{a}^{2} f(u(t-\tau(t)))+T
\end{aligned}
$$

For the drive system (6), the response system is considered as

$$
\begin{aligned}
\frac{d \hat{u}(t)}{d t}= & -A \hat{u}(t)+\hat{b}(t)+v_{1}(t) \\
\frac{d \hat{b}(t)}{d t}= & -B_{a} \hat{b}(t)-C_{a} \hat{u}(t)+\alpha(t) W_{a}^{1} \hat{f}(\hat{u}(t)) \\
& +\beta(t) W_{a}^{2} \hat{f}(\hat{u}(t-\tau(t)))+T+v_{2}(t),
\end{aligned}
$$

where $\hat{u}(t)=\left[\hat{u}_{1}(t), \hat{u}_{2}(t), \ldots, \hat{u}_{n}(t)\right]^{T}, \quad \hat{b}(t)=$ $\left[\hat{b}_{1}(t), \hat{b}_{2}(t), \ldots, \hat{b}_{n}(t)\right]^{T}$ are state responses of controlled system. $v_{1}(t) \in \mathcal{R}^{n}, v_{2}(t) \in \mathcal{R}^{n}$ are control inputs. The errors are $e_{1}(t)=\hat{u}(t)-u(t), e_{2}(t)=\hat{b}(t)-b(t)$, and $g\left(e_{1}(t)\right)=\hat{f}(\hat{u}(t))-f(u(t))$. Then, the error dynamic system is given as follows

$$
\begin{aligned}
\frac{d e_{1}(t)}{d t}= & -A e_{1}(t)+e_{2}(t)+v_{1}(t) \\
\frac{d e_{2}(t)}{d t}= & -B_{a} e_{2}(t)-C_{a} e_{1}(t)+\alpha(t) W_{a}^{1} g\left(e_{1}(t)\right) \\
& +\beta(t) W_{a}^{2} g\left(e_{1}(t-\tau(t))\right)+v_{2}(t) .
\end{aligned}
$$

The control inputs of the error dynamic system (8) are given as follows

$$
\begin{aligned}
& v_{1}(t)=L_{1 a} e_{1}(t), \\
& v_{2}(t)=L_{2 a} e_{2}(t) .
\end{aligned}
$$

Then, system (8) is rewritten as the following form

$$
\begin{aligned}
\frac{d e_{1}(t)}{d t}= & -\left(A-L_{1 a}\right) e_{1}(t)+e_{2}(t), \\
\frac{d e_{2}(t)}{d t}= & -\left(B_{a}-L_{2 a}\right) e_{2}(t)-C_{a} e_{1}(t)+\alpha(t) W_{a}^{1} g\left(e_{1}(t)\right) \\
& +\beta(t) W_{a}^{2} g\left(e_{1}(t-\tau(t))\right) .
\end{aligned}
$$

Lemma 1: ( [49]). For the real scalar $\alpha \in(0,1)$, vectors $\omega_{1}, \omega_{2}$, given symmetric matrices $W_{1}>0, W_{2}>0$, if a matrix $X \in \mathcal{R}^{n \times n}$ satisfies $\left[\begin{array}{cc}W_{1} & X \\ * & W_{2}\end{array}\right] \geq 0$, then the following inequality holds

$$
\begin{aligned}
& \frac{1}{\alpha} \omega_{1}^{T} W_{1} \omega_{1}+\frac{1}{1-\alpha} \omega_{2}^{T} W_{2} \omega_{2} \\
& \geq\left[\begin{array}{c}
\omega_{1} \\
\omega_{2}
\end{array}\right]^{T}\left[\begin{array}{cc}
W_{1} & X \\
* & W_{2}
\end{array}\right]\left[\begin{array}{l}
\omega_{1} \\
\omega_{2}
\end{array}\right] .
\end{aligned}
$$

Lemma 2: (HOPRII, [50]). $x(t)$ is a differentiable function in $\left[a_{1}, a_{2}\right] \rightarrow \mathcal{R}^{n}$ for a time-varying scalar $a(t) \in\left[a_{1}, a_{2}\right]$. For symmetric matrices $Z_{l}=Z_{l}^{T}>0,(l=1,2)$, and any matrices $N_{1}, N_{2}$, the following inequality holds

$$
a_{12} \int_{a_{1}}^{a(t)} \dot{x}^{T}(s) Z_{1} \dot{x}(s) d s+a_{12} \int_{a(t)}^{a_{2}} \dot{x}^{T}(s) Z_{2} \dot{x}(s) d s \geq
$$




$$
\varsigma^{T}(t)\left[\begin{array}{l}
\omega_{1} \\
\omega_{2}
\end{array}\right]^{T} \Delta\left[\begin{array}{l}
\omega_{1} \\
\omega_{2}
\end{array}\right] \varsigma(t)
$$

where

$$
\begin{aligned}
& \Delta=\left[\begin{array}{cc}
\widetilde{Z_{1}}+(1-\gamma) \widetilde{\mho}_{1} & (1-\gamma) N_{1}+\gamma N_{2} \\
* & \widetilde{Z}_{2}+\gamma \widetilde{\mho}_{2}
\end{array}\right], \\
& \gamma=\frac{a(t)-a_{1}}{a_{12}}, a_{12}=a_{2}-a_{1}, \\
& \varsigma(t)=\left[x^{T}\left(a_{2}\right), x^{T}(a(t)), x^{T}\left(a_{1}\right), \varsigma_{1}^{T}(t), \varsigma_{2}^{T}(t), \varsigma_{3}^{T}(t),\right. \\
& \left.\varsigma_{4}^{T}(t), \varsigma_{5}^{T}(t), \varsigma_{6}^{T}(t)\right]^{T}, \\
& \varsigma_{1}(t)=\frac{1}{a(t)-a_{1}} \int_{a_{1}}^{a(t)} x(s) d s, \\
& \varsigma_{2}(t)=\frac{1}{a_{2}-a(t)} \int_{a(t)}^{a_{2}} x(s) d s, \\
& \varsigma_{3}(t)=\frac{2}{\left(a(t)-a_{1}\right)^{2}} \int_{a_{1}}^{a(t)} \int_{u}^{a(t)} x(s) d s d u \\
& \varsigma_{4}(t)=\frac{2}{\left(a_{2}-a(t)\right)^{2}} \int_{a(t)}^{a_{2}} \int_{u}^{a_{2}} x(s) d s d u, \\
& \varsigma_{5}(t)=\frac{6}{\left(a(t)-a_{1}\right)^{3}} \int_{a_{1}}^{a(t)} \int_{u}^{a(t)} \int_{v}^{a(t)} x(s) d s d v d u, \\
& \varsigma_{6}(t)=\frac{6}{\left(a_{2}-a(t)\right)^{3}} \int_{a(t)}^{a_{2}} \int_{u}^{a_{2}} \int_{v}^{a_{2}} x(s) d s d v d u, \\
& \widetilde{Z}_{1}=\operatorname{diag}\left\{Z_{1}, 3 Z_{1}, 5 Z_{1}, 7 Z_{1}\right\}, \widetilde{Z}_{2}=\operatorname{diag}\left\{Z_{2}, 3 Z_{2}, 5 Z_{2}, 7 Z_{2}\right\} \text {, } \\
& \widetilde{\mho}_{1}=\widetilde{Z}_{1}-N_{2} \widetilde{Z}_{2}^{-1} N_{2}^{T}, \widetilde{\mho}_{2}=\widetilde{Z}_{2}-N_{1}^{T} \widetilde{Z}_{1}^{-1} N_{1} \text {, } \\
& \omega_{1}=\left[\left(\widetilde{e}_{2}-\widetilde{e}_{3}\right)^{T},\left(\widetilde{e}_{2}+\widetilde{e}_{3}-2 \widetilde{e}_{4}\right)^{T},\left(\widetilde{e}_{2}-\widetilde{e}_{3}+6 \widetilde{e}_{4}-6 \widetilde{e}_{6}\right)^{T}\right. \text {, } \\
& \left.\left(\widetilde{e}_{2}+\widetilde{e}_{3}-12 \widetilde{e}_{4}+30 \widetilde{e}_{6}-20 \widetilde{e}_{8}\right)^{T}\right]^{T}, \\
& \omega_{2}=\left[\left(\widetilde{e}_{1}-\widetilde{e}_{2}\right)^{T},\left(\widetilde{e}_{1}+\widetilde{e}_{2}-2 \widetilde{e}_{5}\right)^{T},\left(\widetilde{e}_{1}-\widetilde{e}_{2}+6 \widetilde{e}_{5}-6 \widetilde{e}_{7}\right)^{T}\right. \text {, } \\
& \left.\left(\widetilde{e}_{1}+\widetilde{e}_{2}-12 \widetilde{e}_{5}+30 \widetilde{e}_{7}-20 \widetilde{e}_{9}\right)^{T}\right]^{T}, \\
& \widetilde{e}_{k}=\left[0_{n \times(k-1) n} I_{n \times n} 0_{n \times(9-k) n}\right],(k=1,2, \cdots, 9) .
\end{aligned}
$$

Remark 3: According to [50], the matrix $\Delta$ in (12) could provide extra freedom to increase the accuracy of estimating the integral terms.

Lemma 3: ( [51]). For the matrix $S>0$, the following inequality holds

$$
\begin{aligned}
& \int_{l_{1}}^{l_{2}} v^{T}(s) S v(s) d s \geq \frac{1}{l_{2}-l_{1}}\left(\int_{l_{1}}^{l_{2}} v(s) d s\right)^{T} S\left(\int_{l_{1}}^{l_{2}} v(s) d s\right) \\
& +\frac{3}{l_{2}-l_{1}} \Xi_{1}^{T} S \Xi_{1}+\frac{5}{l_{2}-l_{1}} \Xi_{2}^{T} S \Xi_{2},
\end{aligned}
$$

where

$$
\begin{aligned}
\Xi_{1} & =\int_{l_{1}}^{l_{2}} v(s) d s-\frac{2}{l_{2}-l_{1}} \int_{l_{1}}^{l_{2}} \int_{u}^{l_{2}} v(s) d s d u, \\
\Xi_{2} & =\int_{l_{1}}^{l_{2}} v(s) d s-\frac{6}{l_{2}-l_{1}} \int_{l_{1}}^{l_{2}} \int_{u}^{l_{2}} v(s) d s d u \\
& +\frac{12}{\left(l_{2}-l_{1}\right)^{2}} \int_{l_{1}}^{l_{2}} \int_{u}^{l_{2}} \int_{v}^{l_{2}} v(s) d s d v d u
\end{aligned}
$$

\section{MAIN RESULTS}

In this section, the synchronization conditions of generally uncertain Markovian INNs are obtained based on DPTLKF and HOPRII.

Theorem 1. Under Assumption 1, the drive system (6) and response system (7) are synchronous if there are any matrices $X_{1} \in \mathcal{R}^{2 n \times 2 n}, X_{2} \in \mathcal{R}^{6 n \times 6 n}, Y_{l} \in \mathcal{R}^{4 n \times 4 n}(l=1,2)$, invertible matrices $\hat{F}_{l}, F_{l} \in \mathcal{R}^{n \times n}(l=1,2)$, symmetric matrices $P_{1} \in \mathcal{R}^{4 n \times 4 n}, P_{v} \in \mathcal{R}^{2 n \times 2 n}, P_{v}>0(v=2,3), Q_{k p}$, $Q_{k q} \in \mathcal{R}^{2 n \times 2 n}(k=3,4), Z_{l}(l=1,2)$, symmetric positive definite matrices $H_{a}, K_{a}, V_{l a b}(l=1,2), R, M_{1} \in \mathcal{R}^{2 n \times 2 n}$, $M_{2} \in \mathcal{R}^{n \times n}$ and $Q_{l} \in \mathcal{R}^{2 n \times 2 n}(l=1,2)$, positive definite diagonal matrices $R_{1}, R_{2}, R_{3}$, such that for any $a \in \mathcal{C}$, the succeeding matrix inequalities are satisfied.

If $a \notin \mathcal{C}_{k}^{a}$,

$$
\begin{aligned}
& H_{b}-H_{a}-V_{1 a b} \leq 0, \forall b \in \mathcal{C}_{k}^{a}, \\
& K_{b}-K_{a}-V_{2 a b} \leq 0, \forall b \in \mathcal{C}_{k}^{a}, \\
& H_{b}-H_{a} \leq 0, \forall b \in \mathcal{C}_{u k}^{a}, b \neq a, \\
& K_{b}-K_{a} \leq 0, \forall b \in \mathcal{C}_{u k}^{a}, b \neq a, \\
& {\left[\begin{array}{cc}
\Xi\left(\mu_{1}, 0\right) & \Sigma_{1}^{T} Y_{2} \\
* & -\widehat{M}_{2}
\end{array}\right]<0,} \\
& {\left[\begin{array}{cc}
\Xi\left(\mu_{1}, \tau\right) & \Sigma_{2}^{T} Y_{1}^{T} \\
* & -\widehat{M}_{2}
\end{array}\right]<0,} \\
& {\left[\begin{array}{cc}
\Xi\left(\mu_{2}, 0\right) & \Sigma_{1}^{T} Y_{2} \\
* & -\widehat{M}_{2}
\end{array}\right]<0,} \\
& {\left[\begin{array}{cc}
\Xi\left(\mu_{2}, \tau\right) & \Sigma_{2}^{T} Y_{1}^{T} \\
* & -\widehat{M}_{2}
\end{array}\right]<0,} \\
& {\left[\begin{array}{cc}
P_{2} & X_{1} \\
* & P_{3}
\end{array}\right] \geq 0,\left[\begin{array}{cc}
\hat{Q}_{3 q} & X_{2} \\
* & \hat{Q}_{4 q}
\end{array}\right] \geq 0,}
\end{aligned}
$$

$\vartheta_{0}>0, \vartheta_{1}>0, Q_{k, l}>0, \digamma_{\sigma, l}>0,(k=3,4, \sigma, l=1,2)$,

where

$\Xi\left(\mu_{1}, 0\right)=\left.\Gamma(\dot{\tau}(t), \tau(t))\right|_{\dot{\tau}(t)=\mu_{1}, \tau(t)=0}+\Gamma_{t}+\Gamma_{a a}$, $\Xi\left(\mu_{2}, 0\right)=\left.\Gamma(\dot{\tau}(t), \tau(t))\right|_{\dot{\tau}(t)=\mu_{2}, \tau(t)=0}+\Gamma_{t}+\Gamma_{a a}$, $\Xi\left(\mu_{1}, \tau\right)=\left.\Gamma(\dot{\tau}(t), \tau(t))\right|_{\dot{\tau}(t)=\mu_{1}, \tau(t)=\tau}+\Gamma_{t}+\Gamma_{a a}$, $\Xi\left(\mu_{2}, \tau\right)=\left.\Gamma(\dot{\tau}(t), \tau(t))\right|_{\dot{\tau}(t)=\mu_{2}, \tau(t)=\tau}+\Gamma_{t}+\Gamma_{a a}$, $\Gamma(\dot{\tau}(t), \tau(t))=\dot{\tau}(t) \phi_{2}^{T} Q_{1} \phi_{2}+\operatorname{Sym}\left\{\left[\tau(t) e_{7}^{T}+(\tau-\right.\right.$ $\left.\left.\tau(t)) e_{8}^{T}\right] R\left(e_{1}-e_{3}\right)\right\}+\dot{\tau}(t)\left[\Pi_{3}^{T} P_{2} \Pi_{3}-\Pi_{4}^{T} P_{3} \Pi_{4}\right]+$ $\operatorname{Sym}\left\{\Pi_{1}^{T} P_{1} \Pi_{2}(t)\right\}+\operatorname{Sym}\left\{\Pi_{3}^{T} P_{2} \Pi_{5}\right\}+\operatorname{Sym}\left\{\Pi_{4}^{T} P_{3} \Pi_{6}\right\}+$ $\Pi_{11}^{T}\left(Q_{3 p}-\tau(t) Q_{3 q}\right) \Pi_{11}-(1-\dot{\tau}(t)) \Pi_{12}^{T}\left(Q_{3 p}-\right.$ $\left.\tau(t) Q_{3 q}\right) \Pi_{12}+(1-\dot{\tau}(t)) \Pi_{12}^{T}\left(Q_{4 p}+(\tau-\tau(t)) Q_{4 q}\right) \Pi_{12}-$ $\Pi_{13}^{T}\left(Q_{4 p}+(\tau-\tau(t)) Q_{4 q}\right) \Pi_{13}-\operatorname{Sym}\left\{\Pi_{7}^{T} \hat{Q}_{3 q} \Pi_{8}+\right.$ $\left.\Pi_{9}^{T} \hat{Q}_{4 q} \Pi_{10}\right\}-\varrho \tau \Pi_{8}^{T} \hat{Q}_{3 q} \Pi_{8}-(1-\varrho) \tau \Pi_{10}^{T} \hat{Q}_{4 q} \Pi_{10}-$ $\frac{1}{\tau}\left[\begin{array}{l}\Pi_{7} \\ \Pi_{9}\end{array}\right]^{T}\left[\begin{array}{cc}\hat{Q}_{3 q} & X_{2} \\ * & \hat{Q}_{4 q}\end{array}\right]\left[\begin{array}{l}\Pi_{7} \\ \Pi_{9}\end{array}\right]-\left[\begin{array}{c}\Sigma_{1} \\ \Sigma_{2}\end{array}\right]^{T} \hat{\psi}_{2}(\varrho, 1-$ $\varrho)\left[\begin{array}{l}\Sigma_{1} \\ \Sigma_{2}\end{array}\right]$,

$\Gamma_{t}=\operatorname{Sym}\left\{e_{1}^{T} H_{a} e_{14}+e_{13}^{T} K_{a} e_{15}\right\}+\phi_{1}^{T} Q_{1} \phi_{1}+e_{1}^{T} Z_{1} e_{1}$ $-e_{2}^{T} Z_{1} e_{2}+e_{2}^{T} Z_{2} e_{2}-e_{3}^{T} Z_{2} e_{3}+\tau^{2} e_{14}^{T} M_{2} e_{14}-\phi_{2}^{T} Q_{1} \phi_{2}$ $+\phi_{1}^{T} Q_{2} \phi_{1}-\phi_{3}^{T} Q_{2} \phi_{3}+\operatorname{Sym}\left\{e_{1}^{T} F_{1} e_{13}-e_{1}^{T} F_{1}\left(A-L_{1 a}\right) e_{1}\right.$ 
$-e_{1}^{T} F_{1} e_{14}-e_{14}^{T} F_{2}\left(A-L_{1 a}\right) e_{1}+e_{14}^{T} F_{2} e_{13}-e_{14}^{T} F_{2} e_{14}$

$-e_{13}^{T} \hat{F}_{1}\left(B_{a}-L_{2 a}\right) e_{13}+e_{13}^{T} \hat{F}_{1} \bar{\alpha} W_{a}^{1} e_{4}+e_{13}^{T} \hat{F}_{1} \bar{\beta} W_{a}^{2} e_{5}$

$-e_{13}^{T} \hat{F}_{1} e_{15}-e_{13}^{T} \hat{F}_{1} C_{a} e_{1}-e_{15}^{T} \hat{F}_{2}\left(B_{a}-L_{2 a}\right) e_{13}+e_{15}^{T} \hat{F}_{2} \bar{\alpha} W_{a}^{1} e_{4}$

$\left.+e_{15}^{T} \hat{F}_{2} \bar{\beta} W_{a}^{2} e_{5}-e_{15}^{T} \hat{F}_{2} e_{15}-e_{15}^{T} \hat{F}_{2} C_{a} e_{1}\right\}+\tau \Pi_{11}^{T} M_{1} \Pi_{11}$

$+\phi_{1}^{T} \Gamma_{1} \phi_{1}+\phi_{2}^{T} \Gamma_{2} \phi_{2}+\phi_{3}^{T} \Gamma_{3} \phi_{3}$,

$\Gamma_{a a}=e_{1}^{T} \sum_{b \in \mathcal{C}_{k}^{a}}\left[\underline{\lambda}_{a b}\left(H_{b}-H_{a}\right)+2 \varpi_{a b} V_{1 a b}\right] e_{1}$

$+e_{13} \sum_{b \in \mathcal{C}_{k}^{a}}\left[\underline{\lambda}_{a b}\left(K_{b}-K_{a}\right)+2 \varpi_{a b} V_{2 a b}\right] e_{13}$,

$\vartheta_{\varrho}=P_{1}+\tau \Phi_{1}^{T}\left(\varrho P_{2}+(1-\varrho) P_{3}\right) \Phi_{1}+\operatorname{Sym}\left\{\Phi_{1}^{T} P_{2} \Phi_{2}+\right.$ $\left.\Phi_{1}^{T} P_{3} \Phi_{3}\right\}+\frac{1}{\tau}\left[\begin{array}{l}\Phi_{2} \\ \Phi_{3}\end{array}\right]^{T}\left[\begin{array}{cc}P_{2} & X_{1} \\ * & P_{3}\end{array}\right]\left[\begin{array}{l}\Phi_{2} \\ \Phi_{3}\end{array}\right]$,

$\vartheta_{0}=\left.\vartheta_{\varrho}\right|_{\tau(t)=0}, \vartheta_{1}=\left.\vartheta_{\varrho}\right|_{\tau(t)=\tau}, \varrho=\frac{\tau(t)}{\tau}$,

$\Phi_{1}=\left[\begin{array}{ll}\hat{e}_{1}^{T} & 0\end{array}\right]^{T}, \quad \Phi_{2}=\left[\begin{array}{ll}0 & \hat{e}_{3}^{T}\end{array}\right]^{T^{T}}, \quad \Phi_{3}=\left[\begin{array}{ll}0 & \hat{e}_{4}^{T}\end{array}\right]^{T}$, $\hat{e}_{m}=\left[\begin{array}{lll}0_{n \times(m-1) n} I_{n \times n} & 0_{n \times(4-m) n}\end{array}\right], m=1,2,3,4$.

$\phi_{1}=\left[e_{1}^{T}, e_{4}^{T}\right], \phi_{2}=\left[e_{2}^{T}, e_{5}^{T}\right], \phi_{3}=\left[e_{3}^{T}, e_{6}^{T}\right]$, $\hat{\psi}_{2}(\varrho, 1-\varrho)=\left[\begin{array}{cc}(2-\varrho) \widehat{M}_{2} & (1-\varrho) Y_{1}+\varrho Y_{2} \\ * & (1+\varrho) \widehat{M}_{2}\end{array}\right]$,

$\widehat{M}_{2}=\operatorname{diag}\left\{M_{2}, 3 M_{2}, 5 M_{2}, 7 M_{2}\right\}$,

$\hat{\Lambda}_{a}=\varpi_{a a}-\sum_{b \in \mathcal{C}_{k}^{a}, b \neq a} \varpi_{a b}$,

$\underline{\lambda}_{a}=-\bar{\lambda}_{a a}-\sum_{b \in \mathcal{C}_{k}^{a}, b \neq a} \lambda_{a b}$

$\underline{\lambda}_{a b}=\tilde{\lambda}_{a b}-\varpi_{a b}, \bar{\lambda}_{a b}=\tilde{\lambda}_{a b}+\varpi_{a b}$,

$\Pi_{1}=\left[e_{1}^{T}, e_{2}^{T}, \tau(t) e_{7}^{T},(\tau-\tau(t)) e_{8}^{T}\right]^{T}$,

$\Pi_{2}(t)=\left[e_{14}^{T},(1-\dot{\tau}(t)) e_{16}^{T}, e_{1}^{T}-(1-\dot{\tau}(t)) e_{2}^{T},(1-\dot{\tau}(t)) e_{2}^{T}-\right.$ $\left.e_{3}^{T}\right]^{T}$,

$\Pi_{3}=\left[e_{1}^{T}, e_{7}^{T}\right]^{T}, \Pi_{4}=\left[e_{1}^{T}, e_{8}^{T}\right]^{T}$,

$\Pi_{5}=\left[\varrho \tau e_{14}^{T}, e_{1}^{T}-(1-\dot{\tau}(t)) e_{2}^{T}-\dot{\tau}(t) e_{7}^{T}\right]^{T}$,

$\Pi_{6}=\left[(1-\varrho) \tau e_{14}^{T},(1-\dot{\tau}(t)) e_{2}^{T}-e_{3}^{T}+\dot{\tau}(t) e_{8}^{T}\right]^{T}$,

$\Pi_{7}=\left[0, e_{1}^{T}-e_{2}^{T}, 0,-e_{1}^{T}-e_{2}^{T}+2 e_{7}^{T}, 0, e_{1}^{T}-e_{2}^{T}+6 e_{7}^{T}-6 e_{9}^{T}\right]^{T}$,

$\Pi_{8}=\left[e_{7}^{T}, 0, e_{7}^{T}-e_{9}^{T}, 0, e_{7}^{T}-3 e_{9}^{T}+2 e_{11}^{T}, 0\right]^{T}$,

$\Pi_{9}=\left[0, e_{2}^{T}-e_{3}^{T}, 0,-e_{2}^{T}-e_{3}^{T}+2 e_{8}^{T}, 0, e_{2}^{T}-e_{3}^{T}+6 e_{8}^{T}-6 e_{10}^{T}\right]^{T}$,

$\Pi_{10}=\left[e_{8}^{T}, 0, e_{8}^{T}-e_{10}^{T}, 0, e_{8}^{T}-3 e_{10}^{T}+2 e_{12}^{T}, 0\right]^{T}$,

$\Pi_{11}=\left[e_{1}^{T}, e_{14}^{T}\right]^{T}, \Pi_{12}=\left[e_{2}^{T}, e_{16}^{T}\right]^{T}, \Pi_{13}=\left[e_{3}^{T}, e_{17}^{T}\right]^{T}$,

$\Sigma_{1}=\left[\left(e_{1}-e_{2}\right)^{T},\left(e_{1}+e_{2}-2 e_{7}\right)^{T},\left(e_{1}-e_{2}+6 e_{7}-\right.\right.$ $\left.\left.6 e_{9}\right)^{T},\left(e_{1}+e_{2}-12 e_{7}+30 e_{9}-20 e_{11}\right)^{T}\right]^{T}$,

$\Sigma_{2}=\left[\left(e_{2}-e_{3}\right)^{T},\left(e_{2}+e_{3}-2 e_{8}\right)^{T},\left(e_{2}-e_{3}+6 e_{8}-\right.\right.$ $\left.\left.6 e_{10}\right)^{T},\left(e_{2}+e_{3}-12 e_{8}+30 e_{10}-20 e_{12}\right)^{T}\right]^{T}$,

$Q_{31}=Q_{3 p}, Q_{41}=Q_{4 p}, Q_{32}=Q_{3 p}-\tau Q_{3 q}$,

$Q_{42}=Q_{4 p}+\tau Q_{4 q}$,

$\digamma_{1,1}=\left.\digamma_{1}\right|_{\dot{\tau}(t)=\mu_{1}}, \digamma_{1,2}=\left.\digamma_{1}\right|_{\dot{\tau}(t)=\mu_{2}}$,

$\digamma_{2,1}=\left.\digamma_{2}\right|_{\dot{\tau}(t)=\mu_{1}}, \digamma_{2,2}=\left.\digamma_{2}\right|_{\dot{\tau}(t)=\mu_{2}}$,

$\digamma_{1}=M_{1}+\dot{\tau}(t) Q_{3 q}+\left[\begin{array}{cc}0 & Z_{1} \\ Z_{1} & 0 \\ 0 & Z_{2} \\ Z_{2} & 0\end{array}\right]$,

$\hat{Q}_{3 q}=\operatorname{diag}\left\{\digamma_{1}, 3 \digamma_{1}, 5 \digamma_{1}\right\}$,

$\hat{Q}_{4 q}=\operatorname{diag}\left\{\digamma_{2}, 3 \digamma_{2}, 5 \digamma_{2}\right\}$,

$\Gamma_{\alpha_{1}}=\left[\begin{array}{cc}-R_{\alpha_{1}} E_{1} & R_{\alpha_{1}} E_{2} \\ * & -R_{\alpha_{1}}\end{array}\right] \quad\left(\alpha_{1}=1,2,3\right)$.

If $a \in \mathcal{C}_{k}^{a}$,

$$
\begin{aligned}
& H_{b}-H_{a}-V_{1 a b} \leq 0, \forall b \in \mathcal{C}, b \neq a, \\
& K_{b}-K_{a}-V_{2 a b} \leq 0, \forall b \in \mathcal{C}, b \neq a,
\end{aligned}
$$

$$
\begin{gathered}
{\left[\begin{array}{cc}
\hat{\Xi}\left(\mu_{1}, 0\right) & \Sigma_{1}^{T} Y_{2} \\
* & -\widehat{M}_{2}
\end{array}\right]<0,} \\
{\left[\begin{array}{cc}
\hat{\Xi}\left(\mu_{1}, \tau\right) & \Sigma_{2}^{T} Y_{1}^{T} \\
* & -\widehat{M}_{2}
\end{array}\right]<0,} \\
{\left[\begin{array}{cc}
\hat{\Xi}\left(\mu_{2}, 0\right) & \Sigma_{1}^{T} Y_{2} \\
* & -\widehat{M}_{2}
\end{array}\right]<0,} \\
{\left[\begin{array}{cc}
\hat{\Xi}\left(\mu_{2}, \tau\right) & \Sigma_{2}^{T} Y_{1}^{T} \\
* & -\widehat{M}_{2}
\end{array}\right]<0,} \\
{\left[\begin{array}{cc}
P_{2} & X_{1} \\
* & P_{3}
\end{array}\right] \geq 0,\left[\begin{array}{cc}
\hat{Q}_{3 q} & X_{2} \\
* & \hat{Q}_{4 q}
\end{array}\right] \geq 0}
\end{gathered}
$$

$\vartheta_{0}>0, \vartheta_{1}>0, Q_{k, l}>0, \digamma_{\sigma, l}>0,(k=3,4, \sigma, l=1,2)$,

where

$\hat{\Xi}\left(\mu_{1}, 0\right)=\left.\Gamma(\dot{\tau}(t), \tau(t))\right|_{\dot{\tau}(t)=\mu_{1}, \tau(t)=0}+\Gamma_{t}+\hat{\Gamma}_{a a}$,

$\hat{\Xi}\left(\mu_{2}, 0\right)=\left.\Gamma(\dot{\tau}(t), \tau(t))\right|_{\dot{\tau}(t)=\mu_{2}, \tau(t)=0}+\Gamma_{t}+\hat{\Gamma}_{a a}$,

$\hat{\Xi}\left(\mu_{1}, \tau\right)=\left.\Gamma(\dot{\tau}(t), \tau(t))\right|_{\dot{\tau}(t)=\mu_{1}, \tau(t)=\tau}+\Gamma_{t}+\hat{\Gamma}_{a a}$,

$\hat{\Xi}\left(\mu_{2}, \tau\right)=\left.\Gamma(\dot{\tau}(t), \tau(t))\right|_{\dot{\tau}(t)=\mu_{2}, \tau(t)=\tau}+\Gamma_{t}+\hat{\Gamma}_{a a}$,

In matrices $\hat{\Xi}\left(\mu_{1}, 0\right), \hat{\Xi}\left(\mu_{2}, 0\right), \hat{\Xi}\left(\mu_{1}, \tau\right)$ and $\hat{\Xi}\left(\mu_{2}, \tau\right)$, only the element $\hat{\Gamma}_{a a}$ is different from $\Gamma_{a a}$. The other elements are the same as the elements in $\Xi\left(\mu_{1}, 0\right), \Xi\left(\mu_{2}, 0\right), \Xi\left(\mu_{1}, \tau\right)$ and $\Xi\left(\mu_{2}, \tau\right)$.

$\hat{\Gamma}_{a a}=e_{1}^{T}\left[\sum_{b \in \mathcal{C}_{k}^{a}, b \neq a}^{\mathcal{M}}\left[\underline{\lambda}_{a b}\left(H_{b}-H_{a}\right)+2 \varpi_{a b} V_{1 a b}\right]\right.$

$\left.+\underline{\lambda}_{a}\left(H_{l}-H_{a}\right)+2 \hat{\Lambda}_{a} V_{1 a l}\right] e_{1}$

$+e_{13}^{T}\left[\sum_{b \in \mathcal{C}_{k}^{a}, b \neq a}^{\mathcal{M}}\left[\underline{\lambda}_{a b}\left(K_{b}-K_{a}\right)+2 \varpi_{a b} V_{2 a b}\right]\right.$

$\left.+\underline{\lambda}_{a}\left(K_{l}-K_{a}\right)+2 \hat{\Lambda}_{a} V_{2 a l}\right] e_{13},\left(l \in \mathcal{C}_{u k}^{a}\right)$.

Proof Consider the DPTLKF of generally uncertain Markovian error INNs as follows

$V(t, a)=\sum_{r=1}^{7} V_{r}(t, a)$,

$V_{1}(t, a)=e_{1}^{T}(t) H_{a} e_{1}(t)+e_{2}^{T}(t) K_{a} e_{2}(t)$,
$V_{2}(t, a)=\int_{t-\tau(t)}^{t} \phi^{T}(s) Q_{1} \phi(s) d s+\int_{t-\tau}^{t} \phi^{T}(s) Q_{2} \phi(s) d s$

$V_{3}(t, a)=\int_{t-\tau}^{t} e_{1}^{T}(s) d s R \int_{t-\tau}^{t} e_{1}(s) d s$,

$V_{4}(t, a)=\zeta_{1}^{T}(t) P_{1} \zeta_{1}(t)+\tau(t) \zeta_{2}^{T}(t) P_{2} \zeta_{2}(t)$ $+(\tau-\tau(t)) \zeta_{3}^{T}(t) P_{3} \zeta_{3}(t)$,

$V_{5}(t, a)=\int_{t-\tau(t)}^{t} \zeta_{4}^{T}(s) Q_{3}(\tau(t)) \zeta_{4}(s) d s$

$+\int_{t-\tau}^{t-\tau(t)} \zeta_{4}^{T}(s) Q_{4}(\tau(t)) \zeta_{4}(s) d s$,

$V_{6}(t, a)=\int_{-\tau}^{0} \int_{t+\theta}^{t} \zeta_{4}^{T}(s) M_{1} \zeta_{4}(s) d s d \theta$,

$V_{7}(t, a)=\tau \int_{-\tau}^{0} \int_{t+\theta}^{t} \dot{e}_{1}^{T}(s) M_{2} \dot{e}_{1}(s) d s d \theta$, 
where

$\phi(t)=\left[e_{1}^{T}(t), g^{T}\left(e_{1}(t)\right)\right]^{T}$,

$\zeta_{1}(t)=\left[e_{1}^{T}(t), e_{1}^{T}(t-\tau(t)), \int_{t-\tau(t)}^{t} e_{1}^{T}(s) d s\right.$,

$\left.\int_{t-\tau}^{t-\tau(t)} e_{1}^{T}(s) d s\right]^{T}$,

$\zeta_{2}(t)=\left[e_{1}^{T}(t), \frac{1}{\tau(t)} \int_{t-\tau(t)}^{t} e_{1}^{T}(s) d s\right]^{T}$,

$\zeta_{3}(t)=\left[e_{1}^{T}(t), \frac{1}{\tau-\tau(t)} \int_{t-\tau}^{t-\tau(t)} e_{1}^{T}(s) d s\right]^{T}$,

$\zeta_{4}(t)=\left[e_{1}^{T}(t), \dot{e}_{1}^{T}(t)\right]^{T}$,

$Q_{3}(\tau(t))=Q_{3 p}-\tau(t) Q_{3 q}, Q_{4}(\tau(t))=Q_{4 p}+(\tau-\tau(t)) Q_{4 q}$.

In order to prove the positive definiteness of the LKF $V(t, a), V_{4}(t, a)$ is rewritten as

$$
\begin{aligned}
V_{4}(t, a)= & \zeta_{1}^{T}(t)\left\{P_{1}+\tau(t)\left(\Phi_{1}+\frac{1}{\tau(t)} \Phi_{2}\right)^{T} P_{2}\left(\Phi_{1}+\frac{1}{\tau(t)} \Phi_{2}\right)\right. \\
& +(\tau-\tau(t))\left(\Phi_{1}+\frac{1}{\tau-\tau(t)} \Phi_{3}\right)^{T} P_{3}\left(\Phi_{1}\right. \\
& \left.\left.+\frac{1}{\tau-\tau(t)} \Phi_{3}\right)\right\} \zeta_{1}(t) \\
= & \zeta_{1}^{T}(t)\left[P_{1}+\tau \Phi_{1}^{T}\left(\varrho P_{2}+(1-\varrho) P_{3}\right) \Phi_{1}\right. \\
& +\operatorname{Sym}\left\{\Phi_{1}^{T} P_{2} \Phi_{2}+\Phi_{1}^{T} P_{3} \Phi_{3}\right\} \\
& \left.+\frac{1}{\tau}\left(\frac{1}{\varrho} \Phi_{2}^{T} P_{2} \Phi_{2}+\frac{1}{1-\varrho} \Phi_{3}^{T} P_{3} \Phi_{3}\right)\right] \zeta_{1}(t),
\end{aligned}
$$

where

$\Phi_{1}=\left[\begin{array}{ll}\hat{e}_{1}^{T} & 0\end{array}\right]^{T}, \Phi_{2}=\left[\begin{array}{ll}0 & \hat{e}_{3}^{T}\end{array}\right]^{T}, \Phi_{3}=\left[\begin{array}{ll}0 & \hat{e}_{4}^{T}\end{array}\right]^{T}, \hat{e}_{m}=$ $\left[0_{n \times(m-1) n} I_{n \times n} 0_{n \times(4-m) n}\right], m=1,2,3,4$.

$P_{2}>0$ and $P_{3}>0$, then by utilizing Lemma 1, for any matrix $X_{1}$ and $\left[\begin{array}{cc}P_{2} & X_{1} \\ * & P_{3}\end{array}\right] \geq 0$, one has

$\frac{1}{\varrho} \Phi_{2}^{T} P_{2} \Phi_{2}+\frac{1}{1-\varrho} \Phi_{3}^{T} P_{3} \Phi_{3} \geq\left[\begin{array}{c}\Phi_{2} \\ \Phi_{3}\end{array}\right]^{T}\left[\begin{array}{cc}P_{2} & X_{1} \\ * & P_{3}\end{array}\right]\left[\begin{array}{l}\Phi_{2} \\ \Phi_{3}\end{array}\right]$

Thus we have

$$
V_{4}(t, a) \geq \zeta_{1}^{T}(t) \vartheta_{\varrho} \zeta_{1}(t),
$$

where

$$
\begin{aligned}
& \vartheta_{\varrho}=P_{1}+\tau \Phi_{1}^{T}\left(\varrho P_{2}+(1-\varrho) P_{3}\right) \Phi_{1}+\operatorname{Sym}\left\{\Phi_{1}^{T} P_{2} \Phi_{2}\right. \\
& \left.+\Phi_{1}^{T} P_{3} \Phi_{3}\right\}+\frac{1}{\tau}\left[\begin{array}{c}
\Phi_{2} \\
\Phi_{3}
\end{array}\right]^{T}\left[\begin{array}{cc}
P_{2} & X_{1} \\
* & P_{3}
\end{array}\right]\left[\begin{array}{c}
\Phi_{2} \\
\Phi_{3}
\end{array}\right] .
\end{aligned}
$$

From $\vartheta_{0}>0$ and $\vartheta_{1}>0$ in (23), we get $V_{4}(t, a)>0$. From $Q_{k, l}>0(k=3,4, l=1,2)$, we get $V_{5}(t, a)>0 . \mathcal{L}$ is defined as the weak infinitesimal operator. Then, calculating the time derivative of $V(t, a)$, one has

$$
\begin{aligned}
& \mathcal{L} V_{1}(t, a) \\
& =2 e_{1}^{T}(t) H_{a} \dot{e}_{1}(t)+2 e_{2}^{T}(t) K_{a} \dot{e}_{2}(t) \\
& +\sum_{b=1}^{\mathcal{M}} \lambda_{a b} e_{1}^{T}(t) H_{b} e_{1}(t)+\sum_{b=1}^{\mathcal{M}} \lambda_{a b} e_{2}^{T}(t) K_{b} e_{2}(t), \\
& \mathcal{L} V_{2}(t, a) \\
& =\phi^{T}(t) Q_{1} \phi(t)-(1-\dot{\tau}(t)) \phi^{T}(t-\tau(t)) Q_{1} \\
& \phi(t-\tau(t))+\phi^{T}(t) Q_{2} \phi(t)-\phi^{T}(t-\tau) Q_{2} \phi(t-\tau), \\
& \mathcal{L} V_{3}(t, a)
\end{aligned}
$$

$$
\begin{aligned}
& =2\left[\tau(t) \times \frac{1}{\tau(t)} \int_{t-\tau(t)}^{t} e_{1}^{T}(s) d s+(\tau-\tau(t))\right. \\
& \left.\times \frac{1}{\tau-\tau(t)} \int_{t-\tau}^{t-\tau(t)} e_{1}^{T}(s) d s\right] R\left(e_{1}(t)-e_{1}(t-\tau)\right) \\
& \mathcal{L} V_{4}(t, a) \\
& =2 \zeta_{1}^{T}(t) P_{1} \dot{\zeta}_{1}(t)+\dot{\tau}(t) \zeta_{2}^{T}(t) P_{2} \zeta_{2}(t)+2 \tau(t) \zeta_{2}^{T}(t) P_{2} \\
& \times \dot{\zeta}_{2}(t)-\dot{\tau}(t) \zeta_{3}^{T}(t) P_{3} \zeta_{3}(t)+2(\tau-\tau(t)) \zeta_{3}^{T}(t) P_{3} \dot{\zeta}_{3}(t)
\end{aligned}
$$

$\mathcal{L} V_{5}(t, a)$

$$
\begin{aligned}
& =\zeta_{4}^{T}(t)\left(Q_{3 p}-\tau(t) Q_{3 q}\right) \zeta_{4}(t)-(1-\dot{\tau}(t)) \zeta_{4}^{T}(t-\tau(t))\left(Q_{3 p}\right. \\
& \left.-\tau(t) Q_{3 q}\right) \zeta_{4}(t-\tau(t))+(1-\dot{\tau}(t)) \zeta_{4}^{T}(t-\tau(t))\left(Q_{4 p}\right. \\
& \left.+(\tau-\tau(t)) Q_{4 q}\right) \zeta_{4}(t-\tau(t))-\zeta_{4}^{T}(t-\tau)\left(Q_{4 p}+(\tau\right. \\
& \left.-\tau(t)) Q_{4 q}\right) \zeta_{4}(t-\tau)-\dot{\tau}(t) \int_{t-\tau(t)}^{t} \zeta_{4}^{T}(s) Q_{3 q} \zeta_{4}(s) d s \\
& -\dot{\tau}(t) \int_{t-\tau}^{t-\tau(t)} \zeta_{4}^{T}(s) Q_{4 q} \zeta_{4}(s) d s
\end{aligned}
$$

$$
\begin{aligned}
& \mathcal{L} V_{6}(t, a) \\
& =\tau \zeta_{4}^{T}(t) M_{1} \zeta_{4}(t)-\int_{t-\tau}^{t} \zeta_{4}^{T}(s) M_{1} \zeta_{4}(s) d s, \\
& \mathcal{L} V_{7}(t, a) \\
& =\tau^{2} \dot{e}_{1}^{T}(t) M_{2} \dot{e}_{1}(t)-\tau \int_{t-\tau}^{t} \dot{e}_{1}^{T}(s) M_{2} \dot{e}_{1}(s) d s,
\end{aligned}
$$

where

$\dot{\zeta}_{1}(t)=\left[\dot{e}_{1}^{T}(t),(1-\dot{\tau}(t)) \dot{e}_{1}^{T}(t-\tau(t)), e_{1}^{T}(t)-(1-\dot{\tau}(t)) e_{1}^{T}(t\right.$ $\left.-\tau(t)),(1-\dot{\tau}(t)) e_{1}^{T}(t-\tau(t))-e_{1}^{T}(t-\tau)\right]^{T}$, $\dot{\zeta}_{2}(t)=\left[\dot{e}_{1}^{T}(t), \frac{1}{\tau(t)} e_{1}^{T}(t)-\frac{(1-\dot{\tau}(t))}{\tau(t)} e_{1}^{T}(t-\tau(t))\right.$ $\left.-\frac{\dot{\tau}(t)}{\tau^{2}(t)} \int_{t-\tau(t)}^{t} e_{1}^{T}(s) d s\right]^{T}$,

$\dot{\zeta}_{3}(t)=\left[\dot{e}_{1}^{T}(t), \frac{(1-\dot{\tau}(t))}{\tau-\tau(t)} e_{1}^{T}(t-\tau(t))-\frac{1}{\tau-\tau(t)} e_{1}^{T}(t-\tau)\right.$ $\left.+\frac{\dot{\tau}(t)}{(\tau-\tau(t))^{2}} \int_{t-\tau}^{t-\tau(t)} e_{1}^{T}(s) d s\right]^{T}$.

By using Lemma 2, one gets

$$
\begin{aligned}
& -\tau \int_{t-\tau}^{t} \dot{e}_{1}^{T}(s) M_{2} \dot{e}_{1}(s) d s \\
& =-\tau \int_{t-\tau(t)}^{t} \dot{e}_{1}^{T}(s) M_{2} \dot{e}_{1}(s) d s-\tau \int_{t-\tau}^{t-\tau(t)} \dot{e}_{1}^{T}(s) M_{2} \dot{e}_{1}(s) d s \\
& \leq-\chi^{T}(t)\left\{\left[\begin{array}{c}
\Sigma_{1} \\
\Sigma_{2}
\end{array}\right]^{T} \hat{\psi}_{1}(\varrho, 1-\varrho)\left[\begin{array}{c}
\Sigma_{1} \\
\Sigma_{2}
\end{array}\right]\right\} \chi(t) \\
& \leq-\chi^{T}(t)\left\{\left[\begin{array}{c}
\Sigma_{1} \\
\Sigma_{2}
\end{array}\right]^{T} \hat{\psi}_{2}(\varrho, 1-\varrho)\left[\begin{array}{c}
\Sigma_{1} \\
\Sigma_{2}
\end{array}\right]\right\} \chi(t) \\
& +\chi^{T}(t)\left[\varrho \Sigma_{2}^{T} Y_{1}^{T} \widehat{M}_{2}^{-1} Y_{1} \Sigma_{2}\right. \\
& \left.+(1-\varrho) \Sigma_{1}^{T} Y_{2} \widehat{M}_{2}^{-1} Y_{2}^{T} \Sigma_{1}\right] \chi(t),
\end{aligned}
$$

where

$\hat{\psi}_{1}(\varrho, 1-\varrho)=\left[\begin{array}{cc}\widehat{M}_{2}+(1-\varrho) \hat{\Pi}_{1} & (1-\varrho) Y_{1}+\varrho Y_{2} \\ * & \widehat{M}_{2}+\varrho \hat{\Pi}_{2}\end{array}\right]$,

$\hat{\Pi}_{1}=\widehat{M}_{2}-Y_{2} \widehat{M}_{2}^{-1} Y_{2}^{T}, \hat{\Pi}_{2}=\widehat{M}_{2}-Y_{1}^{T} \widehat{M}_{2}^{-1} Y_{1}$,

$\widehat{M}_{2}, \hat{\psi}_{2}(\varrho, 1-\varrho), \varrho, \Sigma_{1}, \Sigma_{2}$ are given in Theorem 1 . 
By using the integration formula, the zero equalities with any symmetric matrices $Z_{l}(l=1,2)$ hold

$$
\begin{aligned}
& 0=e_{1}^{T}(t) Z_{1} e_{1}(t)-e_{1}^{T}(t-\tau(t)) Z_{1} e_{1}(t-\tau(t)) \\
& -2 \int_{t-\tau(t)}^{t} e_{1}^{T}(s) Z_{1} \dot{e}_{1}(s) d s \\
& 0=e_{1}^{T}(t-\tau(t)) Z_{2} e_{1}(t-\tau(t))-e_{1}^{T}(t-\tau) Z_{2} e_{1}(t-\tau) \\
& -2 \int_{t-\tau}^{t-\tau(t)} e_{1}^{T}(s) Z_{2} \dot{e}_{1}(s) d s
\end{aligned}
$$

From (48), one gets

$$
\begin{aligned}
& -\int_{t-\tau}^{t} \zeta_{4}^{T}(s) M_{1} \zeta_{4}(s) d s \\
& =-\int_{t-\tau}^{t-\tau(t)} \zeta_{4}^{T}(s) M_{1} \zeta_{4}(s) d s-\int_{t-\tau(t)}^{t} \zeta_{4}^{T}(s) M_{1} \zeta_{4}(s) d s .
\end{aligned}
$$
we get

Considering single integral terms in (47), (48) and (53),

$$
\begin{aligned}
& \Delta_{1} \\
& =-\int_{t-\tau(t)}^{t} \zeta_{4}^{T}(s)\left(M_{1}+\dot{\tau}(t) Q_{3 q}+\left[\begin{array}{cc}
0 & Z_{1} \\
Z_{1} & 0
\end{array}\right]\right) \zeta_{4}(s) d s,
\end{aligned}
$$

$$
\begin{aligned}
& \Delta_{2} \\
& =-\int_{t-\tau}^{t-\tau(t)} \zeta_{4}^{T}(s)\left(M_{1}+\dot{\tau}(t) Q_{4 q}+\left[\begin{array}{cc}
0 & Z_{2} \\
Z_{2} & 0
\end{array}\right]\right) \zeta_{4}(s) d s .
\end{aligned}
$$

By using Lemma 3, the upper bounds of (54) and (55) are obtained as follows

$$
\begin{aligned}
& \Delta_{1}+\Delta_{2} \\
& \leq-\chi^{T}(t)\left\{\frac{1}{\tau(t)}\left(\Pi_{7}+\tau(t) \Pi_{8}\right)^{T} \hat{Q}_{3 q}\left(\Pi_{7}+\tau(t) \Pi_{8}\right)\right. \\
& +\frac{1}{\tau-\tau(t)}\left(\Pi_{9}+(\tau-\tau(t)) \Pi_{10}\right)^{T} \hat{Q}_{4 q}\left(\Pi_{9}\right. \\
& \left.\left.+(\tau-\tau(t)) \Pi_{10}\right)\right\} \chi(t) \\
& =-\chi^{T}(t)\left\{\operatorname{Sym}\left\{\Pi_{7}^{T} \hat{Q}_{3 q} \Pi_{8}+\Pi_{9}^{T} \hat{Q}_{4 q} \Pi_{10}\right\}+\varrho \tau \Pi_{8}^{T} \hat{Q}_{3 q} \Pi_{8}\right. \\
& +(1-\varrho) \tau \Pi_{10}^{T} \hat{Q}_{4 q} \Pi_{10}+\frac{1}{\tau}\left(\frac{1}{\varrho} \Pi_{7}^{T} \hat{Q}_{3 q} \Pi_{7}\right. \\
& \left.\left.+\frac{1}{1-\varrho} \Pi_{9}^{T} \hat{Q}_{4 q} \Pi_{9}\right)\right\} \chi(t)
\end{aligned}
$$
obtained

By using Lemma 1 and (56), the following inequality is $\Delta_{1}+\Delta_{2}$

$$
\leq-\chi^{T}(t)\left\{\operatorname{Sym}\left\{\Pi_{7}^{T} \hat{Q}_{3 q} \Pi_{8}+\Pi_{9}^{T} \hat{Q}_{4 q} \Pi_{10}\right\}+\varrho \tau \Pi_{8}^{T} \hat{Q}_{3 q} \Pi_{8}\right.
$$$$
+(1-\varrho) \tau \Pi_{10}^{T} \hat{Q}_{4 q} \Pi_{10}+\frac{1}{\tau}\left[\begin{array}{c}
\Pi_{7} \\
\Pi_{9}
\end{array}\right]^{T}\left[\begin{array}{cc}
\hat{Q}_{3 q} & X_{2} \\
* & \hat{Q}_{4 q}
\end{array}\right]
$$$$
\left.\times\left[\begin{array}{l}
\Pi_{7} \\
\Pi_{9}
\end{array}\right]\right\} \chi(t) .
$$

From Assumption 1, one gets

$$
\phi^{T}(t)\left[\begin{array}{cc}
-R_{1} E_{1} & R_{1} E_{2} \\
* & -R_{1}
\end{array}\right] \phi(t) \geq 0,
$$

$$
\begin{aligned}
& \phi^{T}(t-\tau(t))\left[\begin{array}{cc}
-R_{2} E_{1} & R_{2} E_{2} \\
* & -R_{2}
\end{array}\right] \phi(t-\tau(t)) \geq 0, \\
& \phi^{T}(t-\tau)\left[\begin{array}{cc}
-R_{3} E_{1} & R_{3} E_{2} \\
* & -R_{3}
\end{array}\right] \phi(t-\tau) \geq 0 .
\end{aligned}
$$

From (10), one gets

$$
\begin{aligned}
& 0=2 \mathcal{E}\left\{[ e _ { 1 } ^ { T } ( t ) F _ { 1 } + \dot { e } _ { 1 } ^ { T } ( t ) F _ { 2 } ] \left[-\left(A-L_{1 a}\right) e_{1}(t)+e_{2}(t)\right.\right. \\
& \left.\left.-\dot{e}_{1}^{T}(t)\right]\right\},
\end{aligned}
$$

$$
\begin{aligned}
& 0=2 \mathcal{E}\left\{[ e _ { 2 } ^ { T } ( t ) \hat { F } _ { 1 } + \dot { e } _ { 2 } ^ { T } ( t ) \hat { F } _ { 2 } ] \left[-\left(B_{a}-L_{2 a}\right) e_{2}(t)-C_{a} e_{1}(t)\right.\right. \\
& \left.\left.+\alpha(t) W_{a}^{1} g\left(e_{1}(t)\right)+\beta(t) W_{a}^{2} g\left(e_{1}(t-\tau(t))\right)-\dot{e}_{2}^{T}(t)\right]\right\}
\end{aligned}
$$

Then combining (32)-(62), we get $\mathcal{E}\{\mathcal{L} V(t, a)\} \leq$ $\mathcal{E}\left\{\chi^{T}(t) \wp(t) \chi(t)\right\}<0$,

where

$$
\begin{aligned}
\wp(s) & =\bar{\psi}(\dot{\tau}(t), \tau(t))+\varrho \Sigma_{2}^{T} Y_{1}^{T} \widehat{M}_{2}^{-1} Y_{1} \Sigma_{2} \\
& +(1-\varrho) \Sigma_{1}^{T} Y_{2} \widehat{M}_{2}^{-1} Y_{2}^{T} \Sigma_{1},
\end{aligned}
$$

$\bar{\psi}(\dot{\tau}(t), \tau(t))$

$=\Gamma_{t}+\Gamma(\dot{\tau}(t), \tau(t))+e_{1}^{T} \sum_{b=1}^{\mathcal{M}} \lambda_{a b} H_{b} e_{1}+e_{13}^{T} \sum_{b=1}^{\mathcal{M}} \lambda_{a b} K_{b} e_{13}$, $\chi(t)=\left[e_{1}^{T}(t), e_{1}^{T}(t-\tau(t)), e_{1}^{T}(t-\tau), g^{T}\left(e_{1}(t)\right)\right.$, $g^{T}\left(e_{1}(t-\tau(t))\right), g^{T}\left(e_{1}(t-\tau)\right), \frac{1}{\tau(t)} \int_{t-\tau(t)}^{t} e_{1}^{T}(s) d s$, $\frac{1}{\tau-\tau(t)} \int_{t-\tau}^{t-\tau(t)} e_{1}^{T}(s) d s, \frac{2}{(\tau(t))^{2}} \int_{t-\tau(t)}^{t} \int_{\theta}^{t} e_{1}^{T}(s) d s d \theta$, $\frac{2}{(\tau-\tau(t))^{2}} \int_{t-\tau}^{t-\tau(t)} \int_{\theta}^{t-\tau(t)} e_{1}^{T}(s) d s d \theta$, $\frac{6}{(\tau(t))^{3}} \int_{t-\tau(t)}^{t} \int_{\theta}^{t} \int_{\alpha}^{t} e_{1}^{T}(s) d s d \alpha d \theta$, $\frac{6}{(\tau-\tau(t))^{3}} \int_{t-\tau}^{t-\tau(t)} \int_{\theta}^{t-\tau(t)} \int_{\alpha}^{t-\tau(t)} e_{1}^{T}(s) d s d \alpha d \theta$, $\left.e_{2}^{T}(t), \dot{e}_{1}^{T}(t), \dot{e}_{2}^{T}(t), \dot{e}_{1}^{T}(t-\tau(t)), \dot{e}_{1}^{T}(t-\tau)\right]^{T}$.

According to the convex theory, $\mathcal{E}\left\{\chi^{T}(t) \wp(t) \chi(t)\right\}<0$ could be satisfied for all $(\dot{\tau}(t), \tau(t)) \in\left[\mu_{1}, \mu_{2}\right] \times[0, \tau]$ if it is satisfied at the vertices of the interval $\left[\mu_{1}, \mu_{2}\right] \times[0, \tau]$.

According to Schur complement, if one gets $\mathcal{E}\left\{\bar{\psi}^{\gamma}\right\}<$ $0(\gamma=1,2,3,4)$, then one gets $\mathcal{E}\left\{\chi^{T}(t) \wp(t) \chi(t)\right\}<0$, where,

$$
\begin{aligned}
\bar{\psi}^{1} & =\left[\begin{array}{cc}
\bar{\psi}\left(\mu_{1}, 0\right) & \Sigma_{1}^{T} Y_{2} \\
* & -\widehat{M}_{2}
\end{array}\right], \bar{\psi}^{2}=\left[\begin{array}{cc}
\bar{\psi}\left(\mu_{1}, \tau\right) & \Sigma_{2}^{T} Y_{1}^{T} \\
* & -\widehat{M}_{2}
\end{array}\right], \\
\bar{\psi}^{3} & =\left[\begin{array}{cc}
\bar{\psi}\left(\mu_{2}, 0\right) & \Sigma_{1}^{T} Y_{2} \\
* & -\widehat{M}_{2}
\end{array}\right], \bar{\psi}^{4}=\left[\begin{array}{cc}
\bar{\psi}\left(\mu_{2}, \tau\right) & \Sigma_{2}^{T} Y_{1}^{T} \\
* & -\widehat{M}_{2}
\end{array}\right] .
\end{aligned}
$$

$\bar{\psi}\left(\mu_{1}, 0\right)=\left.\bar{\psi}(\dot{\tau}(t), \tau(t))\right|_{\dot{\tau}(t)=\mu_{1}, \tau(t)=0}$, $\bar{\psi}\left(\mu_{2}, 0\right)=\left.\bar{\psi}(\dot{\tau}(t), \tau(t))\right|_{\dot{\tau}(t)=\mu_{2}, \tau(t)=0}$, $\bar{\psi}\left(\mu_{1}, \tau\right)=\left.\bar{\psi}(\dot{\tau}(t), \tau(t))\right|_{\dot{\tau}(t)=\mu_{1}, \tau(t)=\tau}$, $\bar{\psi}\left(\mu_{2}, \tau\right)=\left.\bar{\psi}(\dot{\tau}(t), \tau(t))\right|_{\dot{\tau}(t)=\mu_{2}, \tau(t)=\tau}$.

Now, we prove that $\mathcal{E}\left\{\bar{\psi}^{\gamma}\right\}<0(\gamma=1,2,3,4)$ hold if (14)-(23) are satisfied. Hence, if $\mathcal{E}\left\{\bar{\psi}^{\gamma}\right\}<0(\gamma=1,2,3,4)$ hold, $\mathcal{E}\left\{\chi^{T}(t) \wp(t) \chi(t)\right\}<0$ is satisfied. Then the error system (10) is globally asymptotically stable in the mean square, and the generally Markovian INNs drive system (6) and response system (7) are synchronous.

Let $\hat{\Upsilon}_{1}^{\gamma}=\hat{\Theta}_{1}^{\gamma}+\sum_{b=1}^{\mathcal{M}} \lambda_{a b} H_{b}, \hat{\Upsilon}_{2}^{\gamma}=\hat{\Theta}_{2}^{\gamma}+\sum_{b=1}^{\mathcal{M}} \lambda_{a b} K_{b}$,

$\hat{\Theta}_{1}^{\gamma}=e_{1}\left(\Gamma_{t}+\bar{\Gamma}^{\gamma}\right) e_{1}^{T}, \hat{\Theta}_{2}^{\gamma}=e_{13}\left(\Gamma_{t}+\bar{\Gamma}^{\gamma}\right) e_{13}^{T},(\gamma=1,2,3,4)$.

$\bar{\Gamma}^{1}=\left.\Gamma(\dot{\tau}(t), \tau(t))\right|_{\dot{\tau}(t)=\mu_{1}, \tau(t)=0}$,

$\bar{\Gamma}^{2}=\left.\Gamma(\dot{\tau}(t), \tau(t))\right|_{\dot{\tau}(t)=\mu_{1}, \tau(t)=\tau}$, 
$\bar{\Gamma}^{3}=\left.\Gamma(\dot{\tau}(t), \tau(t))\right|_{\dot{\tau}(t)=\mu_{2}, \tau(t)=0}$,

$\bar{\Gamma}^{4}=\left.\Gamma(\dot{\tau}(t), \tau(t))\right|_{\dot{\tau}(t)=\mu_{2}, \tau(t)=\tau}$.

If $a \notin \mathcal{C}_{k}^{a}$, one gets $\sum_{b=1}^{\mathcal{M}} \lambda_{a b} H_{b}=\sum_{b \in \mathcal{C}_{k}^{a}} \lambda_{a b} H_{b}+\lambda_{a a} H_{a}$

$+\sum_{b \in \mathcal{C}_{u k}^{a}, b \neq a} \lambda_{a b} H_{b}$, where $\lambda_{a b} \geq 0, b \neq a$ and $0 \leq$ $\sum_{b \in \mathcal{C}_{u k}^{a}, b \neq a} \lambda_{a b}=-\lambda_{a a}-\sum_{b \in \mathcal{C}_{k}^{a}} \lambda_{a b}$.

From $H_{b}-H_{a}-V_{1 a b} \leq 0\left(\forall b \in \mathcal{C}_{k}^{a}\right)$ in (14) and $H_{b}-$ $H_{a} \leq 0\left(\forall b \in \mathcal{C}_{u k}^{a}, b \neq a\right)$ in (16), one has

$$
\begin{aligned}
& \sum_{b \in \mathcal{C}_{k}^{a}} \lambda_{a b} H_{b}+\lambda_{a a} H_{a}+\sum_{b \in \mathcal{C}_{u k}^{a}, b \neq a} \lambda_{a b} H_{b} \\
\leq & \sum_{b \in \mathcal{C}_{k}^{a}} \lambda_{a b} H_{b}+\lambda_{a a} H_{a}+\sum_{b \in \mathcal{C}_{u k}^{a}, b \neq a} \lambda_{a b} H_{a} \\
= & \sum_{b \in \mathcal{C}_{k}^{a}} \lambda_{a b} H_{b}+\lambda_{a a} H_{a}+\left(-\lambda_{a a}-\sum_{b \in \mathcal{C}_{k}^{a}} \lambda_{a b}\right) H_{a} \\
= & \sum_{b \in \mathcal{C}_{k}^{a}} \lambda_{a b}\left(H_{b}-H_{a}\right) \\
= & \sum_{b \in \mathcal{C}_{k}^{a}}\left(\underline{\lambda}_{a b}+\varpi_{a b}+\triangle_{a b}\right)\left(H_{b}-H_{a}\right) \\
= & \sum_{b \in \mathcal{C}_{k}^{a}}\left[\underline{\lambda}_{a b}\left(H_{b}-H_{a}\right)+\left(\varpi_{a b}+\triangle_{a b}\right)\left(H_{b}-H_{a}\right)\right] .
\end{aligned}
$$

Noting that $V_{1 a b} \geq 0, \triangle_{a b} \in\left[-\varpi_{a b}, \varpi_{a b}\right], \forall b \in \mathcal{C}_{k}^{a}$, and $H_{b}-H_{a} \leq 0, \forall b \in \mathcal{C}_{u k}^{a}, b \neq a$, one has

$\left(\varpi_{a b}+\triangle_{a b}\right)\left(H_{b}-H_{a}\right) \leq\left(\varpi_{a b}+\triangle_{a b}\right) V_{1 a b} \leq 2 \varpi_{a b} V_{1 a b}, \forall b \in \mathcal{C}_{k}^{a}$.

Hence, one gets

$$
\hat{\Upsilon}_{1}^{\gamma} \leq \hat{\Theta}_{1}^{\gamma}+\sum_{b \in \mathcal{C}_{k}^{a}}\left(\underline{\lambda}_{a b}\left(H_{b}-H_{a}\right)+2 \varpi_{a b} V_{1 a b}\right) .
$$

Similarly, for $K_{b}-K_{a}-V_{2 a b} \leq 0\left(\forall b \in \mathcal{C}_{k}^{a}\right)$ in (15) and $K_{b}-K_{a} \leq 0\left(\forall b \in \mathcal{C}_{u k}^{a}, b \neq a\right)$ in (17), one has

$$
\hat{\Upsilon}_{2}^{\gamma} \leq \hat{\Theta}_{2}^{\gamma}+\sum_{b \in \mathcal{C}_{k}^{a}}\left(\underline{\lambda}_{a b}\left(K_{b}-K_{a}\right)+2 \varpi_{a b} V_{2 a b}\right) .
$$

Hence, from (14)-(23), one gets $\mathcal{E}\left\{\bar{\psi}^{\gamma}\right\}<0,(\gamma=1,2,3,4)$.

If $a \in \mathcal{C}_{k}^{a}$, according to $\lambda_{a a}=-\sum_{b \in \mathcal{C}, b \neq a} \lambda_{a b}$, there is $H_{b} \leq H_{l}$, for $b, l \in \mathcal{C}_{u k}^{a}$, one has

$$
\begin{aligned}
& \sum_{b=1}^{\mathcal{M}} \lambda_{a b} H_{b} \\
& =\sum_{b \in \mathcal{C}_{k}^{a}, b \neq a} \lambda_{a b} H_{b}+\sum_{b \in \mathcal{C}_{u k}^{a}} \lambda_{a b} H_{b}+\lambda_{a a} H_{a} \\
& =\sum_{b \in \mathcal{C}_{k}^{a}, b \neq a} \lambda_{a b}\left(H_{b}-H_{a}\right)+\sum_{b \in \mathcal{C}_{u k}^{a}} \lambda_{a b}\left(H_{b}-H_{a}\right) \\
& \leq \sum_{b \in \mathcal{C}_{k}^{a}, b \neq a} \lambda_{a b}\left(H_{b}-H_{a}\right)+\sum_{b \in \mathcal{C}_{u k}^{a}} \lambda_{a b}\left(H_{l}-H_{a}\right),\left(l \in \mathcal{C}_{u k}^{a}\right) .
\end{aligned}
$$

From $H_{b}-H_{a}-V_{1 a b} \leq 0(\forall b \in \mathcal{C}, b \neq a)$ in (24), one has

$$
\begin{aligned}
& \sum_{b \in \mathcal{C}_{k}^{a}, b \neq a} \lambda_{a b}\left(H_{b}-H_{a}\right) \\
\leq & \sum_{b \in \mathcal{C}_{k}^{a}, b \neq a}\left[\underline{\lambda}_{a b}\left(H_{b}-H_{a}\right)+2 \varpi_{a b} V_{1 a b}\right], \\
& \sum_{b \in \mathcal{C}_{u k}^{a}} \lambda_{a b}\left(H_{l}-H_{a}\right) \\
\leq & \sum_{b \in \mathcal{C}_{u k}^{a}}\left[\underline{\lambda}_{a b}\left(H_{l}-H_{a}\right)+2 \varpi_{a b} V_{1 a l}\right] .
\end{aligned}
$$

From $\lambda_{a a}=-\sum_{b \in \mathcal{C}_{k}^{a}, b \neq a} \lambda_{a b}-\sum_{b \in \mathcal{C}_{u k}^{a}} \lambda_{a b}$, one gets

$$
\begin{aligned}
& \sum_{b \in \mathcal{C}_{u k}^{a}} \underline{\lambda}_{a b}=-\bar{\lambda}_{a a}-\sum_{b \in \mathcal{C}_{k}^{a}, b \neq a} \underline{\lambda}_{a b}=\underline{\lambda}_{a}, \\
& \sum_{b \in \mathcal{C}_{u k}^{a}} \bar{\lambda}_{a b}=-\underline{\lambda}_{a a}-\sum_{b \in \mathcal{C}_{k}^{a}, b \neq a} \bar{\lambda}_{a b} .
\end{aligned}
$$

Then, we have

$$
\begin{aligned}
& \sum_{b \in \mathcal{C}_{u k}^{a}} \varpi_{a b} \\
& =\frac{1}{2}\left(-\sum_{b \in \mathcal{C}_{u k}^{a}} \underline{\lambda}_{a b}+\sum_{b \in \mathcal{C}_{u k}^{a}} \bar{\lambda}_{a b}\right) \\
& =\frac{1}{2}\left(\bar{\lambda}_{a a}+\sum_{b \in \mathcal{C}_{k}^{a}, b \neq a} \underline{\lambda}_{a b}-\underline{\lambda}_{a a}-\sum_{b \in \mathcal{C}_{k}^{a}, b \neq a} \bar{\lambda}_{a b}\right) \\
& =\varpi_{a a}-\sum_{b \in \mathcal{C}_{k}^{a}, b \neq a} \varpi_{a b} \\
& =\hat{\Lambda}_{a} .
\end{aligned}
$$

Thus, we get

$$
\sum_{b \in \mathcal{C}_{u k}^{a}} \lambda_{a b}\left(H_{b}-H_{a}\right) \leq \underline{\lambda}_{a}\left(H_{l}-H_{a}\right)+2 \hat{\Lambda}_{a} V_{1 a l} .
$$

Hence, one gets

$$
\begin{aligned}
& \hat{\Upsilon}_{1}^{\gamma} \leq \hat{\Theta}_{1}^{\gamma}+\underline{\lambda}_{a}\left(H_{l}-H_{a}\right)+2 \hat{\Lambda}_{a} V_{1 a l} \\
& +\sum_{b \in \mathcal{C}_{k}^{a}, b \neq a}\left(\underline{\lambda}_{a b}\left(H_{b}-H_{a}\right)+2 \varpi_{a b} V_{1 a b}\right) .
\end{aligned}
$$

Similarly, for $K_{b}-K_{a}-V_{2 a b} \leq 0(\forall b \in \mathcal{C}, b \neq a)$ in (25), one has

$$
\begin{aligned}
& \hat{\Upsilon}_{2}^{\gamma} \leq \hat{\Theta}_{2}^{\gamma}+\underline{\lambda}_{a}\left(K_{l}-K_{a}\right)+2 \hat{\Lambda}_{a} V_{2 a l} \\
& +\sum_{b \in \mathcal{C}_{k}^{a}, b \neq a}\left(\underline{\lambda}_{a b}\left(K_{b}-K_{a}\right)+2 \varpi_{a b} V_{2 a b}\right) .
\end{aligned}
$$

Hence, from (24) to (31), one gets $\mathcal{E}\left\{\bar{\psi}^{\gamma}\right\}<0(\gamma=1,2,3,4)$.

Remark 4: In most existing generally uncertain Markovian neural networks [27], [45], [46], Schur complement and matrix inequality of Lemma 2 in [52] are adopted to deal with the estimate error $\triangle_{a b}$ and the completely unknown transition rate "?" in the generally uncertain TRM. Different from these papers, only one set of relaxation variables are adopted in this paper to deal with generally uncertain rates of INNS, 
which reduces the dimension and computational complexity of synchronization conditions.

Remark 5: The DPTLKF in (32) and HOPRII are applied in this paper to obtain the new synchronization of generally uncertain INNS, and the new delay-range-dependent synchronization conditions including more information about timevarying delay and its derivative are proposed in Theorem 1.

Remark 6: From the viewpoint of practical application, the Lyapunov functions contain more information about INNs system, which could reduce the conservativeness of the synchronization conditions and obtain better controllers of the system.

In Theorem 1, the synchronization problem of Markovian inertial neural networks is investigated. Since matrices $F_{l}, \hat{F}_{l}$, and $L_{l a}(l=1,2)$ are not given, the matrix inequalities are nonlinear. Hence, the desired controllers could not be directly solved. According to [26], [53], the nonlinear matrix inequalities are converted into linear matrix inequalities in Theorem 2 , and the corresponding controllers could be obtained.

Theorem 2. Under Assumption 1, the given scalars $\rho_{1}, \rho_{2}$, the drive system (6) and response system (7) are synchronous if there are any matrices $X_{1} \in \mathcal{R}^{2 n \times 2 n}, X_{2} \in$ $\mathcal{R}^{6 n \times 6 n}, Y_{l} \in \mathcal{R}^{4 n \times 4 n}(l=1,2), J_{l a} \in \mathcal{R}^{n \times n}(l=1,2)$, any invertible matrices $\mathcal{F}, \hat{\mathcal{F}}$, symmetric matrices $P_{1} \in \mathcal{R}^{4 n \times 4 n}$, $P_{v} \in \mathcal{R}^{2 n \times 2 n}, P_{v}>0(v=2,3), Q_{k p}, Q_{k q} \in \mathcal{R}^{2 n \times 2 n}(k=$ $3,4), Z_{l}(l=1,2)$, symmetric positive definite matrices $H_{a}$, $K_{a}, V_{l a b}(l=1,2), R, M_{1} \in \mathcal{R}^{2 n \times 2 n}, M_{2} \in \mathcal{R}^{n \times n}$ and $Q_{l} \in \mathcal{R}^{2 n \times 2 n}(l=1,2)$, positive definite diagonal matrices $R_{1}, R_{2}, R_{3}$ such that, for any $a \in \mathcal{C}$, the succeeding linear matrix inequalities are satisfied.

$$
\text { If } a \notin \mathcal{C}_{k}^{a} \text {, }
$$

$$
\begin{aligned}
& H_{b}-H_{a}-V_{1 a b} \leq 0, \forall b \in \mathcal{C}_{k}^{a}, \\
& K_{b}-K_{a}-V_{2 a b} \leq 0, \forall b \in \mathcal{C}_{k}^{a} \text {, } \\
& H_{b}-H_{a} \leq 0, \forall b \in \mathcal{C}_{u k}^{a}, b \neq a, \\
& K_{b}-K_{a} \leq 0, \forall b \in \mathcal{C}_{u k}^{a}, b \neq a, \\
& {\left[\begin{array}{cc}
\tilde{\Xi}\left(\mu_{1}, 0\right) & \Sigma_{1}^{T} Y_{2} \\
* & -\widehat{M}_{2}
\end{array}\right]<0,} \\
& {\left[\begin{array}{cc}
\tilde{\Xi}\left(\mu_{1}, \tau\right) & \Sigma_{2}^{T} Y_{1}^{T} \\
* & -\widehat{M}_{2}
\end{array}\right]<0,} \\
& {\left[\begin{array}{cc}
\tilde{\Xi}\left(\mu_{2}, 0\right) & \Sigma_{1}^{T} Y_{2} \\
* & -\widehat{M}_{2}
\end{array}\right]<0,} \\
& {\left[\begin{array}{cc}
\tilde{\Xi}\left(\mu_{2}, \tau\right) & \Sigma_{2}^{T} Y_{1}^{T} \\
* & -\widehat{M}_{2}
\end{array}\right]<0,} \\
& {\left[\begin{array}{cc}
P_{2} & X_{1} \\
* & P_{3}
\end{array}\right] \geq 0,\left[\begin{array}{cc}
\hat{Q}_{3 q} & X_{2} \\
* & \hat{Q}_{4 q}
\end{array}\right] \geq 0}
\end{aligned}
$$

$\vartheta_{0}>0, \vartheta_{1}>0, Q_{k, l}>0, \digamma_{\sigma, l}>0(k=3,4, \sigma, l=1,2)$,

where

$$
\begin{aligned}
& \tilde{\Xi}\left(\mu_{1}, 0\right)=\left.\Gamma(\dot{\tau}(t), \tau(t))\right|_{\dot{\tau}(t)=\mu_{1}, \tau(t)=0}+\tilde{\Gamma}_{t}+\Gamma_{a a}, \\
& \tilde{\Xi}\left(\mu_{2}, 0\right)=\left.\Gamma(\dot{\tau}(t), \tau(t))\right|_{\dot{\tau}(t)=\mu_{2}, \tau(t)=0}+\tilde{\Gamma}_{t}+\Gamma_{a a},
\end{aligned}
$$

$$
\begin{aligned}
& \tilde{\Xi}\left(\mu_{1}, \tau\right)=\left.\Gamma(\dot{\tau}(t), \tau(t))\right|_{\dot{\tau}(t)=\mu_{1}, \tau(t)=\tau}+\tilde{\Gamma}_{t}+\Gamma_{a a}, \\
& \tilde{\Xi}\left(\mu_{2}, \tau\right)=\left.\Gamma(\dot{\tau}(t), \tau(t))\right|_{\dot{\tau}(t)=\mu_{2}, \tau(t)=\tau}+\tilde{\Gamma}_{t}+\Gamma_{a a}, \\
& \tilde{\Gamma}_{t}=\operatorname{Sym}\left\{e_{1}^{T} H_{a} e_{14}+e_{13}^{T} K_{a} e_{15}\right\}+\phi_{1}^{T} Q_{1} \phi_{1}+e_{1}^{T} Z_{1} e_{1} \\
& -e_{2}^{T} Z_{1} e_{2}+e_{2}^{T} Z_{2} e_{2}-e_{3}^{T} Z_{2} e_{3}+\tau^{2} e_{14}^{T} M_{2} e_{14}-\phi_{2}^{T} Q_{1} \phi_{2} \\
& +\phi_{1}^{T} Q_{2} \phi_{1}-\phi_{3}^{T} Q_{2} \phi_{3}+\operatorname{Sym}\left\{e_{1}^{T} \mathcal{F} e_{13}-e_{1}^{T} \mathcal{F} A e_{1}-e_{1}^{T} \mathcal{F} e_{14}\right. \\
& -\rho_{1} e_{14}^{T} \mathcal{F} A e_{1}+\rho_{1} e_{14}^{T} \mathcal{F} e_{13}-\rho_{1} e_{14}^{T} \mathcal{F} e_{14}-e_{13}^{T} \hat{\mathcal{F}} B_{a} e_{13} \\
& +e_{13}^{T} \hat{\mathcal{F}} \bar{\alpha} W_{a}^{1} e_{4}+e_{13}^{T} \hat{\mathcal{F}} \bar{\beta} W_{a}^{2} e_{5}-e_{13}^{T} \hat{\mathcal{F}} e_{15}-e_{13}^{T} \hat{\mathcal{F}} C_{a} e_{1} \\
& -\rho_{2} e_{15}^{T} \hat{\mathcal{F}} B_{a} e_{13}+\rho_{2} e_{15}^{T} \hat{\mathcal{F}} \bar{\alpha} W_{a}^{1} e_{4}+\rho_{2} e_{15}^{T} \hat{\mathcal{F}} \bar{\beta} W_{a}^{2} e_{5} \\
& -\rho_{2} e_{15}^{T} \hat{\mathcal{F}} e_{15}-\rho_{2} e_{15}^{T} \hat{\mathcal{F}} C_{a} e_{1}+e_{1}^{T} J_{1 a} e_{1}+\rho_{1} e_{14}^{T} J_{1 a} e_{1} \\
& \left.+e_{13}^{T} J_{2 a} e_{13}+\rho_{2} e_{15}^{T} J_{2 a} e_{13}\right\}+\tau \Pi_{11}^{T} M_{1} \Pi_{11}+\phi_{1}^{T} \Gamma_{1} \phi_{1} \\
& +\phi_{2}^{T} \Gamma_{2} \phi_{2}+\phi_{3}^{T} \Gamma_{3} \phi_{3},
\end{aligned}
$$

In matrices $\tilde{\Xi}\left(\mu_{1}, 0\right), \tilde{\Xi}\left(\mu_{1}, \tau\right), \tilde{\Xi}\left(\mu_{2}, 0\right)$ and $\tilde{\Xi}\left(\mu_{2}, \tau\right)$, only element $\tilde{\Gamma}_{t}$ is different from $\Gamma_{t}$ in Theorem 1 . The other elements are the same as the elements in Theorem 1.

If $a \in \mathcal{C}_{k}^{a}$,

$$
\begin{aligned}
& H_{b}-H_{a}-V_{1 a b} \leq 0, \forall b \in \mathcal{C}, b \neq a, \\
& K_{b}-K_{a}-V_{2 a b} \leq 0, \forall b \in \mathcal{C}, b \neq a,
\end{aligned}
$$

$$
\left[\begin{array}{cc}
P_{2} & X_{1} \\
* & P_{3}
\end{array}\right] \geq 0,\left[\begin{array}{cc}
\hat{Q}_{3 q} & X_{2} \\
* & \hat{Q}_{4 q}
\end{array}\right] \geq 0
$$

$\vartheta_{0}>0, \vartheta_{1}>0, Q_{k, l}>0, \digamma_{\sigma, l}>0,(k=3,4, \sigma, l=1,2)$,

$\breve{\Xi}\left(\mu_{1}, 0\right)=\left.\Gamma(\dot{\tau}(t), \tau(t))\right|_{\dot{\tau}(t)=\mu_{1}, \tau(t)=0}+\tilde{\Gamma}_{t}+\hat{\Gamma}_{a a}$,
$\breve{\Xi}\left(\mu_{2}, 0\right)=\left.\Gamma(\dot{\tau}(t), \tau(t))\right|_{\dot{\tau}(t)=\mu_{2}, \tau(t)=0}+\tilde{\Gamma}_{t}+\hat{\Gamma}_{a a}$,
$\breve{\Xi}\left(\mu_{1}, \tau\right)=\left.\Gamma(\dot{\tau}(t), \tau(t))\right|_{\dot{\tau}(t)=\mu_{1}, \tau(t)=\tau}+\tilde{\Gamma}_{t}+\hat{\Gamma}_{a a}$,
$\breve{\Xi}\left(\mu_{2}, \tau\right)=\left.\Gamma(\dot{\tau}(t), \tau(t))\right|_{\dot{\tau}(t)=\mu_{2}, \tau(t)=\tau}+\tilde{\Gamma}_{t}+\hat{\Gamma}_{a a}$,

In matrices $\breve{\Xi}\left(\mu_{1}, 0\right), \breve{\Xi}\left(\mu_{1}, \tau\right), \breve{\Xi}\left(\mu_{2}, 0\right)$, and $\breve{\Xi}\left(\mu_{2}, \tau\right)$, only element $\hat{\Gamma}_{a a}$ is different from $\Gamma_{a a}$. The other elements are the same as the elements in $\tilde{\Xi}\left(\mu_{1}, 0\right), \tilde{\Xi}\left(\mu_{1}, \tau\right), \tilde{\Xi}\left(\mu_{2}, 0\right)$, and $\tilde{\Xi}\left(\mu_{2}, \tau\right)$.

$\hat{\Gamma}_{a a}=e_{1}^{T}\left[\sum_{b \in \mathcal{C}_{k}^{a}, b \neq a}^{\mathcal{M}}\left[\underline{\lambda}_{a b}\left(H_{b}-H_{a}\right)+2 \varpi_{a b} V_{1 a b}\right]\right.$

$\left.+\underline{\lambda}_{a}\left(H_{l}-H_{a}\right)+2 \hat{\Lambda}_{a} V_{1 a l}\right] e_{1}$

$+e_{13}^{T}\left[\sum_{b \in \mathcal{C}_{k}^{a}, b \neq a}^{\mathcal{M}}\left[\underline{\lambda}_{a b}\left(K_{b}-K_{a}\right)+2 \varpi_{a b} V_{2 a b}\right]\right.$

$\left.+\underline{\lambda}_{a}\left(K_{l}-K_{a}\right)+2 \hat{\Lambda}_{a} V_{2 a l}\right] e_{13}, \quad\left(l \in \mathcal{C}_{u k}^{a}\right)$. 
Moreover, the desired controller gain matrices are given as follows

$$
\begin{aligned}
& L_{1 a}=\mathcal{F}^{-1} J_{1 a} \\
& L_{2 a}=\hat{\mathcal{F}}^{-1} J_{2 a}
\end{aligned}
$$

Proof Assume $F_{1}=\mathcal{F}, F_{2}=\rho_{1} F_{1}, \hat{F}_{1}=\hat{\mathcal{F}}, \hat{F}_{2}=\rho_{2} \hat{F}_{1}$ and $\mathcal{F} L_{1 a}=J_{1 a}, \hat{\mathcal{F}} L_{2 a}=J_{2 a}$. From Theorem 1, (78)-(97) hold. The proof is completed.

In Theorem 2, the nonlinear matrix inequalities of synchronization conditions in Theorem 1 are changed into linear matrix inequalities (LMIs). By adopting LMI toolbox in Matlab, the controllers of INNs system could be obtained from Theorem 2.

\section{Simulation}

In this section, two examples are shown to demonstrate the effectiveness of the proposed method.

Example 1. Consider the INNs system as follows

$$
\begin{aligned}
& \frac{d^{2} u_{1}(t)}{d t^{2}}=-a_{1}\left(\imath_{t}\right) \frac{d u_{1}(t)}{d t}-b_{1}\left(\imath_{t}\right) u_{1}(t)+\alpha_{1}(t) w_{11}^{1}\left(\imath_{t}\right) f_{1}\left(u_{1}(t)\right) \\
& +\beta_{1}(t) w_{12}^{1}\left(\imath_{t}\right) f_{2}\left(u_{2}(t)\right)+\alpha(t) w_{11}^{2}\left(\imath_{t}\right) f_{1}\left(u_{1}(t-\tau(t))\right)+\beta(t) \\
& w_{12}^{2}\left(\imath_{t}\right) f_{2}\left(u_{2}(t-\tau(t))\right)+T_{1} \\
& \frac{d^{2} u_{2}(t)}{d t^{2}}=-a_{2}\left(\imath_{t}\right) \frac{d u_{2}(t)}{d t}-b_{2}\left(\imath_{t}\right) u_{2}(t)+\alpha(t) w_{21}^{1}\left(\imath_{t}\right) f_{1}\left(u_{1}(t)\right) \\
& +\beta(t) w_{22}^{1}\left(\imath_{t}\right) f_{2}\left(u_{2}(t)\right)+\alpha(t) w_{21}^{2}\left(\imath_{t}\right) f_{1}\left(u_{1}(t-\tau(t))\right)+\beta(t) \\
& w_{22}^{2}\left(\imath_{t}\right) f_{2}\left(u_{2}(t-\tau(t))\right)+T_{2}
\end{aligned}
$$

The activation functions $f_{k}\left(u_{k}(t)\right)=\tanh \left(u_{k}(t)\right)(k=1,2)$ satisfy Assumption 1. We get $E_{1}=\left(\begin{array}{ll}0 & 0 \\ 0 & 0\end{array}\right), E_{2}=$ $\left(\begin{array}{cc}0.5 & 0 \\ 0 & 0.5\end{array}\right)$.

The system (98) is considered with the following parameters

$$
\begin{aligned}
& A=\left(\begin{array}{ll}
1 & 0 \\
0 & 1
\end{array}\right) \text {, } \\
& B\left(\imath_{t}\right)=\left(\begin{array}{cc}
a_{1}\left(\imath_{t}\right)-\xi_{1} & 0 \\
0 & a_{2}\left(\imath_{t}\right)-\xi_{2}
\end{array}\right), \quad C\left(\imath_{t}\right)= \\
& \left(\begin{array}{cc}
b_{1}\left(\imath_{t}\right)+\xi_{1}\left(\xi_{1}-a_{1}\left(\imath_{t}\right)\right) & 0 \\
0 & b_{2}\left(\imath_{t}\right)+\xi_{2}\left(\xi_{2}-a_{2}\left(\imath_{t}\right)\right)
\end{array}\right), \\
& \left(\imath_{t}=1,2,3\right), B_{1}=\left(\begin{array}{cc}
1.0 & 0 \\
0 & 1.5
\end{array}\right), B_{2}=\left(\begin{array}{cc}
1.6 & 0 \\
0 & 1.2
\end{array}\right), \\
& B_{3}=\left(\begin{array}{cc}
1.2 & 0 \\
0 & 0.9
\end{array}\right), \quad C_{1}=\left(\begin{array}{cc}
-1.1 & 0 \\
0 & -0.9
\end{array}\right) \text {, } \\
& C_{2}=\left(\begin{array}{cc}
-0.7 & 0 \\
0 & -1.0
\end{array}\right), C_{3}=\left(\begin{array}{cc}
-1.0 & 0 \\
0 & -1.1
\end{array}\right) \text {, } \\
& W_{1}^{1}=\left(\begin{array}{cc}
0.9 & 0.2 \\
-0.5 & 2.7
\end{array}\right), W_{2}^{1}=\left(\begin{array}{cc}
-1.0 & 1.7 \\
-1.0 & 1.3
\end{array}\right), \\
& W_{3}^{1}=\left(\begin{array}{ll}
-1.0 & 1.4 \\
-1.0 & 1.0
\end{array}\right) \text {, } \\
& W_{1}^{2}=\left(\begin{array}{cc}
0.9 & 2.1 \\
-3.2 & 0.8
\end{array}\right), W_{2}^{2}=\left(\begin{array}{cc}
-1.7 & 0.8 \\
-1.2 & 1.3
\end{array}\right), W_{3}^{2}= \\
& \left(\begin{array}{ll}
-1.2 & 1.3 \\
-1.2 & 0.3
\end{array}\right), \mho_{1}=\mho_{2}=1, \bar{\alpha}=0.01, \bar{\beta}=0.02, \nu_{\alpha}= \\
& 0.002, \nu_{\beta}=0.004, \tau(t)=0.8+0.2 \sin (t), \tau=1.0, \mu_{1}=
\end{aligned}
$$

$-0.2, \mu_{2}=0.2$. The transition matrix is given as

$\Omega=\left(\begin{array}{ccc}? & ? & 2.32+\triangle_{13} \\ ? & -4.75+\triangle_{22} & 2.02+\Delta_{23} \\ 1.34+\triangle_{31} & ? & -5.05+\triangle_{33}\end{array}\right)$,

where $\varpi_{13}=0.1, \varpi_{22}=\varpi_{23}=0.19, \varpi_{31}=\varpi_{33}=0.18$.

Set the parameters $\rho_{1}=\rho_{2}=1$. From Theorem 2, one gets the following feasible matrices.

$$
\begin{aligned}
& L_{11}=\left(\begin{array}{cc}
-5.9326 & -0.0033 \\
-0.0025 & -6.1008
\end{array}\right), L_{12}= \\
& \left(\begin{array}{cc}
-5.7784 & -0.0042 \\
-0.0023 & -6.1008
\end{array}\right), L_{13}=\left(\begin{array}{ll}
-5.9842 & -0.0033 \\
-0.0027 & -6.2532
\end{array}\right), \\
& L_{21}=\left(\begin{array}{cc}
-3.4484 & 0.0030 \\
-0.0001 & -2.8959
\end{array}\right), L_{22}= \\
& \left(\begin{array}{cc}
-2.7404 & 0.0021 \\
-0.0003 & -3.1412
\end{array}\right), L_{23}=\left(\begin{array}{ll}
0.0961 & -0.0006 \\
0.0001 & -0.2162
\end{array}\right) .
\end{aligned}
$$

The trajectories of error system with initial values $e_{1}(t)=$ $\left[\begin{array}{l}0.3 \\ 0.1\end{array}\right], e_{2}(t)=\left[\begin{array}{c}0.18 \\ -0.22\end{array}\right]$ are given in Figs. 1-2.

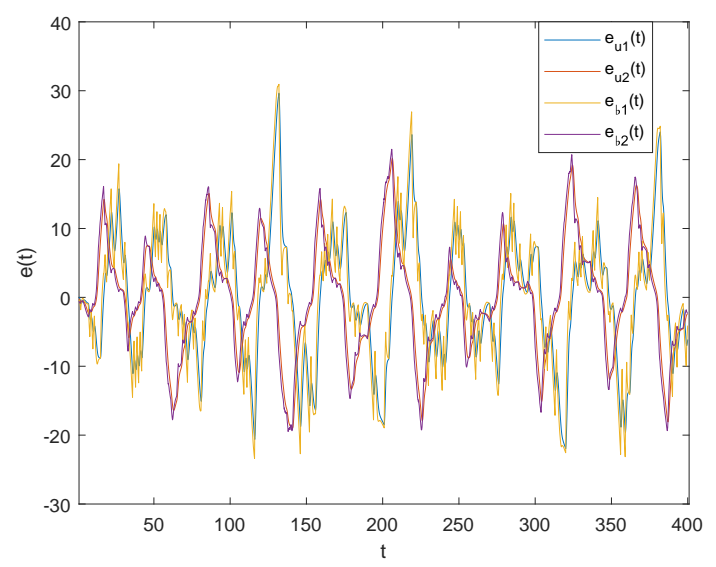

Fig. 1 State trajectories of error system without controllers.

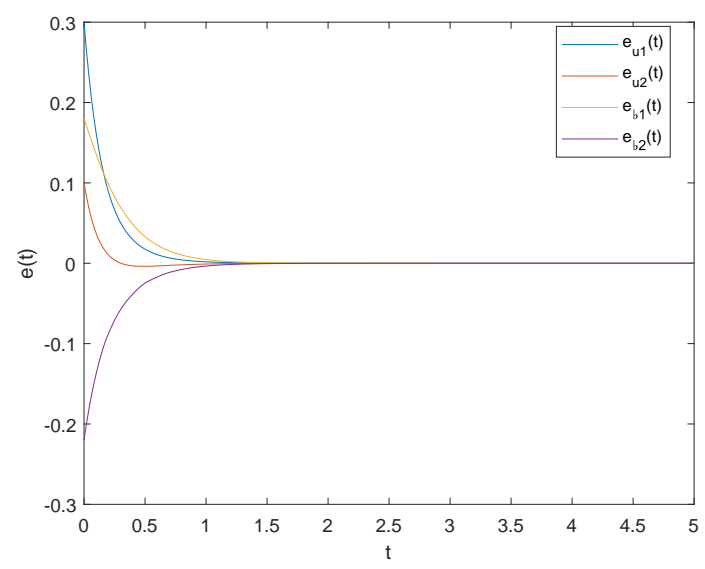

Fig. 2 State trajectories of error system with controllers.

Through the Theorem 2 and Figs. 1-2, one gets that the error system is asymptotically stable. So the driven system and response system could be synchronous under the designed controllers.

Example 2. Under the obtained results, the image with a size $L \times H \times 3$ could be encrypted. According to [44], the process of image encryption is shown as follows. 
Input: A color image $F_{O}$ with the size of $L \times H \times 3$.

Output: An encrypted image $E$ with the size of $L \times H$.

S1: By separating color image $F_{O}$ with red, green, and blue components, respectively, pixel series are obtained as $R(i, j), G(i, j), B(i, j)(i \in\{1, \cdots, L\}, j \in\{1, \cdots, H\})$.

S2: Under uncontrolled system (6), by adopting fourth order Runge-Kutta method with step size 0.001 and the initial conditions $u(0), b(0), \hat{u}(0), \hat{b}(0)$ with more than $I=L \times H$ times iterations, we could get a group of time series chaotic signals.

S3: Master system is iterated $I$ times continuously. For each iteration, we get four values $u, b, \hat{u}, \hat{b}$. Hence, four floating-point number sequences of $X_{1}, X_{2}, X_{3}, X_{4}$ with the length of $I=L \times H$ are obtained as follows:

$$
\begin{aligned}
& X_{1}=\left\{x_{1}(1), x_{1}(2), \cdots, x_{1}(I)\right\}, \\
& X_{2}=\left\{x_{2}(1), x_{2}(2), \cdots, x_{2}(I)\right\}, \\
& X_{3}=\left\{x_{3}(1), x_{3}(2), \cdots, x_{3}(I)\right\}, \\
& X_{4}=\left\{x_{4}(1), x_{4}(2), \cdots, x_{4}(I)\right\} .
\end{aligned}
$$

S4: From these sequences, $X_{R}, X_{G}$ and $X_{B}$ are generated from $X_{k}(k=1,2,3,4)$.

$x_{R}(i)=\left(\left|x_{1}(i)\right|-\left\lfloor\left|x_{1}(i)\right|\right\rfloor\right) \times 10^{14} \bmod 256$

$x_{G}(i)=\left(\left|x_{2}(i)\right|-\left\lfloor\left|x_{2}(i)\right|\right\rfloor\right) \times 10^{14} \bmod 256$

$x_{B}(i)=\left(\left|x_{3}(i)+x_{4}(i)\right|-\left\lfloor\left|x_{3}(i)+x_{4}(i)\right|\right\rfloor\right) \times 10^{14} \bmod 256$

where $x_{R}(i) \in X_{R}, x_{G}(i) \in X_{G}$ and $x_{B} \in X_{B}(i=$ $1, \cdots, I) .\lfloor\rfloor$ represents the values of nearest integer that is less than or equal to $X$. The $\bmod (\cdot, \cdot)$ represents the remainder after division. The size of each sequence $\left(x_{i}\right)$ is $I$.

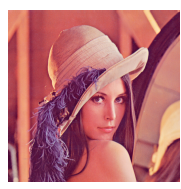

(a) Lena.

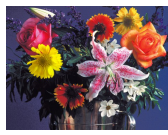

(e) Flowers.

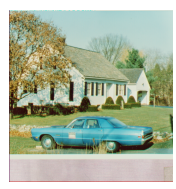

(b) House.

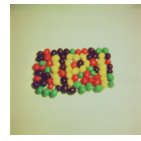

(f) Jelly.

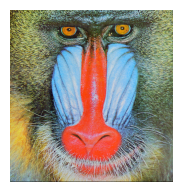

(c) Mandrill.

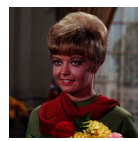

(g) Lady.

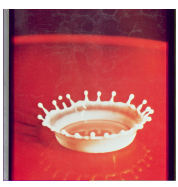

(d) Splash.

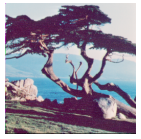

(h) Tree. process of color image is shown as Figs. 3-9. The entropy values of original and encrypted images in Fig. 3 are shown in Table I. The obtained values of the encrypted images are very close to the theoretical value 8 . According to [44], [54], [55], the encrypted image in this paper approaches a random source, and information leakage from the encryption algorithm is negligible.

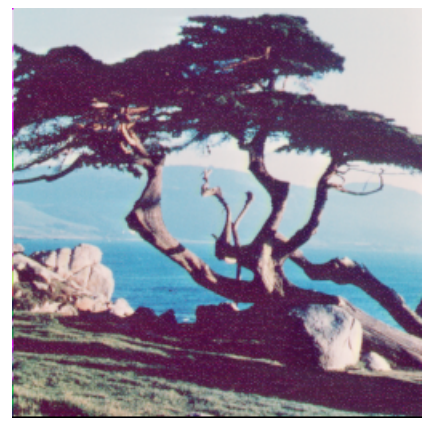

Fig. 4 The original image of the tree.
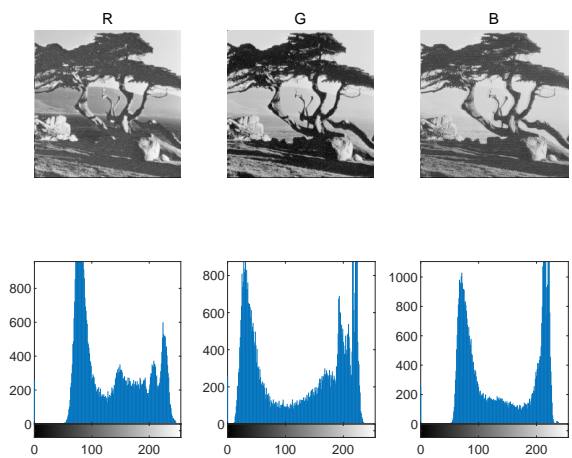

Fig. 5 Histogram of the RGB components of the tree.
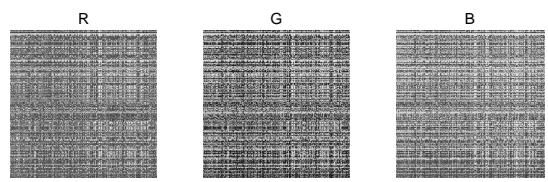

Fig. 3 The original images.

5: The RGB components of $F_{S}$ are encrypted by $X_{R}, X_{B}$ and $X_{G}$, and $e_{R}, e_{B}$ and $e_{G}$ of the encrypted image could be obtained as follows.

$$
\begin{aligned}
& e_{R}(i)=f_{R}(i) \oplus x_{R}(i) \\
& e_{G}(i)=f_{G}(i) \oplus x_{G}(i) \\
& e_{B}(i)=f_{B}(i) \oplus x_{B}(i)
\end{aligned}
$$

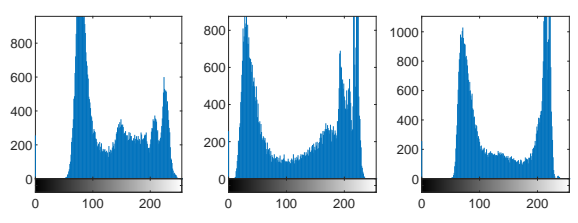

where $\oplus$ is the bitwise $X O R$ operator. $f_{R}(i), f_{G}(i)$ and $f_{B}(i)$ are the pixel sequence of the shuffled image. The encryption
Fig. 6 Histogram of the RGB components of the shuffled image of the tree. 


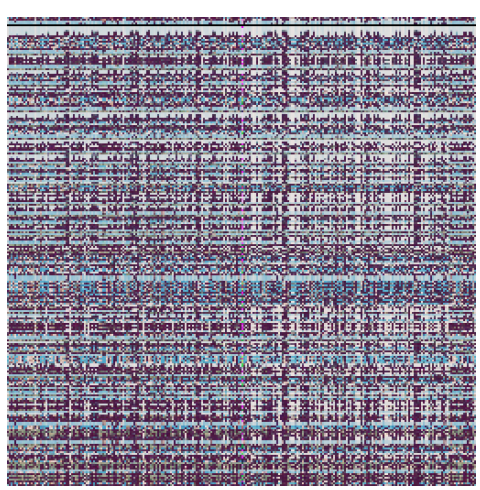

Fig. 7 The shuffled image of the tree.
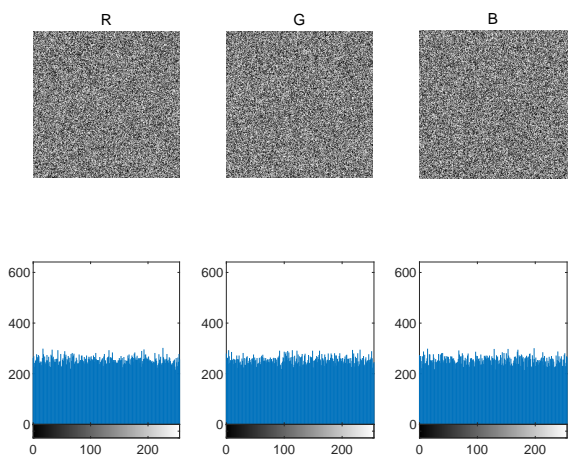

Fig. 8 Histogram of the RGB components of the encrypted image of the tree.
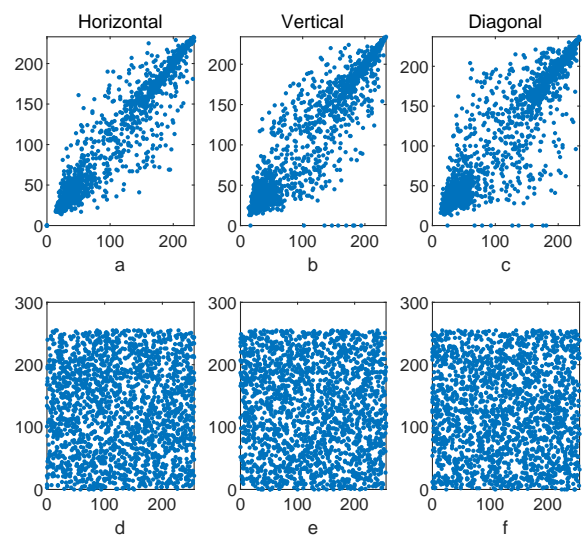

Fig. 9 Correlations of two adjacent pixels in horizontal, vertical, and diagonal directions of the red component of the tree. The figures a-c denote the original image, and d-f denote encrypted image.

$\mathrm{CC}$ is the correlations between original image and encrypted image in [44], [54], [55]. The correlation between two adjacent pixels is shown in Table II. According to the $\mathrm{CC}$ values, the proposed method in this paper has gained good performance. The original and encrypted images are significantly different because the $\mathrm{CC}$ values of the encrypted
TABLE I

ENTROPY ANALYSIS OF RGB COMPONENTS

\begin{tabular}{|c|c|c|c|c|c|c|c|}
\hline Image & Size & \multicolumn{3}{|c|}{ Original Image } & \multicolumn{3}{|c|}{ Encrypted Image } \\
\hline & & Red & Green & Blue & Red & Green & Blue \\
\hline Lena & $512 \times 512$ & 7.2531 & 7.5940 & 6.9684 & 7.9992 & 7.9994 & 7.9993 \\
\hline House & $512 \times 512$ & 7.4156 & 7.2295 & 7.4354 & 7.9993 & 7.9993 & 7.9992 \\
\hline Mandrill & $512 \times 512$ & 7.7067 & 7.4744 & 7.7522 & 7.9992 & 7.9993 & 7.9994 \\
\hline Splash & $512 \times 512$ & 6.9481 & 6.8845 & 6.1265 & 7.9992 & 7.9994 & 7.9993 \\
\hline Flowers & $362 \times 500$ & 7.3824 & 7.2345 & 7.3641 & 7.9989 & 7.9990 & 7.9989 \\
\hline Jelly & $256 \times 256$ & 5.2626 & 5.6947 & 6.5464 & 7.9973 & 7.9970 & 7.9913 \\
\hline Lady & $256 \times 256$ & 6.4200 & 6.4457 & 6.3807 & 7.9972 & 7.9968 & 7.9973 \\
\hline Tree & $256 \times 256$ & 7.2104 & 7.4136 & 6.9207 & 7.9976 & 7.9973 & 7.9969 \\
\hline
\end{tabular}

\begin{tabular}{cccccccccc}
\hline Image & \multicolumn{3}{c}{ Red } & \multicolumn{3}{c}{ Green } & \multicolumn{3}{c}{ Blue } \\
\hline & \multicolumn{3}{c}{$\mathrm{H}$} & $\mathrm{V}$ & $\mathrm{D}$ & $\mathrm{H}$ & $\mathrm{V}$ & $\mathrm{D}$ & \multicolumn{2}{c}{$\mathrm{H}$} & $\mathrm{V}$ & $\mathrm{D}$ \\
\hline Original Lena & 0.9798 & 0.9893 & 0.9697 & 0.9327 & 0.9576 & 0.9183 & 0.9327 & 0.9576 & 0.9183 \\
\hline Encrypted Lena & 0.0016 & -0.0035 & 0.0019 & 0.0015 & -0.0011 & -0.0008 & -0.0011 & 0.0000 & 0.0001 \\
\hline Original House & 0.9536 & 0.9579 & 0.9224 & 0.9725 & 0.9686 & 0.9445 & 0.9391 & 0.9423 & 0.8901 \\
\hline Encrypted House & 0.0028 & -0.0004 & -0.0024 & -0.0008 & 0.0009 & -0.0004 & 0.0011 & 0.0013 & 0.0023 \\
\hline Original Mandrill & 0.9231 & 0.8660 & 0.8543 & 0.9073 & 0.8809 & 0.8399 & 0.8655 & 0.7650 & 0.7348 \\
\hline Encrypted Mandrill & 0.0030 & -0.0007 & 0.0029 & -0.0005 & 0.0031 & 0.0014 & 0.0018 & -0.0039 & -0.0019 \\
\hline Original Splash & 0.9936 & 0.9951 & 0.9894 & 0.9826 & 0.9789 & 0.9649 & 0.9812 & 0.9871 & 0.9711 \\
\hline Encrypted Splash & -0.0011 & -0.0050 & -0.0032 & -0.0007 & 0.0038 & -0.0017 & -0.0021 & 0.0036 & 0.0017 \\
\hline Original Flowers & 0.9718 & 0.9719 & 0.9551 & 0.9527 & 0.9527 & 0.9256 & 0.9510 & 0.9497 & 0.9218 \\
\hline Encrypted Flowers & 0.0001 & 0.0004 & -0.0020 & -0.0005 & 0.0003 & 0.0026 & 0.0051 & 0.0012 & -0.0066 \\
\hline Original Jelly & 0.9745 & 0.9763 & 0.9537 & 0.9890 & 0.9880 & 0.9799 & 0.9757 & 0.9801 & 0.9603 \\
\hline Encrypted Jelly & 0.0063 & -0.0065 & -0.0024 & -0.0020 & -0.0003 & -0.0026 & 0.0009 & 0.0019 & 0.0045 \\
\hline Original Lady & 0.9729 & 0.9622 & 0.9482 & 0.9584 & 0.9519 & 0.9377 & 0.9719 & 0.9647 & 0.9500 \\
\hline Encrypted Lady & -0.0010 & -0.0046 & 0.0019 & 0.0002 & 0.0035 & -0.0033 & 0.0027 & -0.0049 & 0.0052 \\
\hline Original Tree & 0.9590 & 0.9361 & 0.9159 & 0.9612 & 0.9406 & 0.9265 & 0.9687 & 0.9457 & 0.9318 \\
\hline Encrypted Tree & 0.0011 & 0.0013 & -0.0041 & 0.0028 & 0.0059 & -0.0018 & 0.0017 & 0.0008 & 0.0001 \\
\hline
\end{tabular}

images in Table II approach to 0 .

\section{CONClusion}

In this paper, the synchronization problem of delayed INNs with generally uncertain Markovian jumping and random connection weight strengths is investigated. By implementing the DPTLKF and HOPRII, the DRDSC and corresponding controllers are obtained in this paper. Finally, two examples including image encryption application are shown to demonstrate the effectiveness of the theoretical results. Further studies include finite-time sampled-data synchronization and impulsive synchronization control of INNs with generally uncertain Markovian jumping and unbounded time delay.

\section{REFERENCES}

[1] A. Michel, J. Farrell, and H.-F. Sun, "Analysis and synthesis techniques for hopfield type synchronous discrete time neural networks with application to associative memory," IEEE Transactions on Circuits and Systems, vol. 37, no. 11, pp. 1356-1366, 1990.

[2] Y. Xia and J. Wang, "A general projection neural network for solving monotone variational inequalities and related optimization problems," IEEE Transactions on Neural Networks, vol. 15, no. 2, pp. 318-328, 2004.

[3] X. Yang, J. Cao, and J. Lu, "Synchronization of Markovian coupled neural networks with nonidentical node-delays and random coupling strengths," IEEE Transactions on Neural Networks and Learning Systems, vol. 23, no. 1, pp. 60-71, 2012.

[4] H. Zhang, Z. Wang, and D. Liu, "A comprehensive review of stability analysis of continuous-time recurrent neural networks," IEEE Transactions on Neural Networks and Learning Systems, vol. 25, no. 7, pp. 1229-1262, 2014.

[5] J. Shi and Z. Zeng, "Global exponential stabilization and lag synchronization control of inertial neural networks with time delays," Neural Networks, vol. 126, pp. 11-20, 2020.

[6] X. Li, D. O'Regan, and H. Akca, "Global exponential stabilization of impulsive neural networks with unbounded continuously distributed delays," IMA Journal of Applied Mathematics, vol. 80, no. 1, pp. 85-99, 2015. 
[7] S. Wen, Z. Zeng, T. Huang, Q. Meng, and W. Yao, "Lag synchronization of switched neural networks via neural activation function and applications in image encryption," IEEE Transactions on Neural Networks and Learning Systems, vol. 26, no. 7, pp. 1493-1502, 2015.

[8] G. Tan, Z. Wang, and Z. Shi, "Proportional-integral state estimator for quaternion-valued neural networks with time-varying delays," IEEE Transactions on Neural Networks and Learning Systems doi: 10.1109/TNNLS.2021.3103979, 2021.

[9] H. Zhang, C. Liu, H. Su, and K. Zhang, "Echo state network-based decentralized control of continuous-time nonlinear large-scale interconnected systems," IEEE Transactions on Systems, Man, and Cybernetics: Systems, vol. 51, no. 10, pp. 6293-6303, 2021.

[10] H. Zhang, Y. Liu, J. Dai, and Y. Wang, "Command filter based adaptive fuzzy finite-time control for a class of uncertain nonlinear systems with hysteresis," IEEE Transactions on Fuzzy Systems, vol. 29, no. 9, pp. 2553-2564, 2021.

[11] D. Peng, X. Li, R. Rakkiyappan, and Y. Ding, "Stabilization of stochastic delayed systems: event-triggered impulsive control," Applied Mathematics and Computation, vol. 401, p. 126054, 2021.

[12] Y. Liu, X. Liu, Y. Jing, X. Chen, and J. Qiu, "Direct adaptive preassigned finite-time control with time-delay and quantized input using neural network," IEEE Transactions on Neural Networks and Learning Systems, vol. 31, no. 4, pp. 1222-1231, 2020.

[13] W. Qi, J. H. Park, G. Zong, J. Cao, and J. Cheng, "Filter for positive stochastic nonlinear switching systems with phase-type semi-Markov parameters and application," IEEE Transactions on Systems, Man, and Cybernetics: Systems, doi: 10.1109/TSMC.2020.3049137.

[14] S. Sun, H. Zhang, J. Zhang, L. Yu, and J. Sun, "Multiple delaydependent robust $H_{\infty}$ finite-time filtering for uncertain Ito stochastic Takagi-Sugeno fuzzy semi-Markovian jump systems with state constraints," IEEE Transactions on Fuzzy Systems, doi: 10.1109/TFUZZ.2020.3037959.

[15] F. Abdollahi and K. Khorasani, "A decentralized Markovian jump $H_{\infty}$ control routing strategy for mobile multi-agent networked systems," IEEE Transactions on Control Systems Technology, vol. 19, no. 2, pp. 269-283, 2011.

[16] W. Qi, G. Zong, and S.-F. Su, "Fault detection for semi-Markov switching systems in the presence of positivity constraints," IEEE Transactions on Cybernetics doi: 10.1109/TCYB.2021.3096948, 2021.

[17] C. Xu, D. Tong, Q. Chen, W. Zhou, and P. Shi, "Exponential stability of Markovian jumping systems via adaptive sliding mode control," IEEE Transactions on Systems, Man, and Cybernetics: Systems, vol. 51, no. 2, pp. 954-964, 2021.

[18] C. Sowmiya, R. Raja, Q. Zhu, and G. Rajchakit, "Further mean-square asymptotic stability of impulsive discrete-time stochastic bam neural networks with Markovian jumping and multiple time-varying delays," Journal of the Franklin Institute, vol. 356, no. 1, pp. 561-591, 2019.

[19] W. Qi, X. Yang, J. H. Park, J. Cao, and J. Cheng, "Fuzzy SM$\mathrm{C}$ for quantized nonlinear stochastic switching systems with semiMarkovian process and application," IEEE Transactions on Cybernetics doi: 10.1109/TCYB.2021.3069423, 2021.

[20] Q. Ma, S. Xu, and Y. Zou, "Stability and synchronization for Markovian jump neural networks with partly unknown transition probabilities," Neurocomputing, vol. 74, no. 17, pp. 3404-3411, 2011.

[21] X. Huang and Y. Ma, "Improved results on synchronisation of Markovian jump complex dynamical networks via sampled-data controller and convex combination," International Journal of Systems Science, vol. 50, no. 15, pp. 2764-2775, 2019.

[22] Y. Guo, "Stability and stabilization of Markov jump systems with generally uncertain transition rates," Journal of the Franklin Institute, vol. 358, no. 3, pp. 1765-1781, 2021.

[23] R. Rakkiyappan, K. Maheswari, G. Velmurugan, and J. H. Park, "Eventtriggered $H_{\infty}$ state estimation for semi-Markov jumping discrete-time neural networks with quantization," Neural Networks, vol. 105, pp. 236248,2018

[24] Z. Wu, H. Su, and J. Chu, "State estimation for discrete Markovian jumping neural networks with time delay," Neurocomputing, vol. 73, no. 10-12, pp. 2247-2254, 2010 .

[25] W. Qi, G. Zong, and W. X. Zheng, "Adaptive event-triggered SMC for stochastic switching systems with semi-Markov process and application to boost converter circuit model," IEEE Transactions on Circuits and Systems I: Regular Papers, vol. 68, no. 2, pp. 786-796, 2021.

[26] J. Wang, H. Zhang, Z. Wang, and Z. Liu, "Sampled-data synchronization of Markovian coupled neural networks with mode delays based on modedependent LKF," IEEE Transactions on Neural Metworks and Learning Systems, vol. 28, no. 11, pp. 2626-2637, 2017.
[27] J. Wang, H. Zhang, Z. Wang, and Q. Shan, "Local synchronization criteria of Markovian nonlinearly coupled neural networks with uncertain and partially unknown transition rates," IEEE Transactions on Systems, Man, and Cybernetics: Systems, vol. 47, no. 8, pp. 1953-1964, 2017.

[28] O. L. Costa and M. D. Fragoso, "Stability results for discrete-time linear systems with Markovian jumping parameters," Journal of mathematical analysis and applications, vol. 179, no. 1, pp. 154-178, 1993.

[29] Z. Wang, Y. Liu, L. Yu, and X. Liu, "Exponential stability of delayed recurrent neural networks with Markovian jumping parameters," Physics Letters A, vol. 356, no. 4-5, pp. 346-352, 2006.

[30] L. Zhang, E.-K. Boukas, and J. Lam, "Analysis and synthesis of Markov jump linear systems with time-varying delays and partially known transition probabilities," IEEE Transactions on Automatic Control, vol. 53, no. 10, pp. 2458-2464, 2008.

[31] Q. Zhu and J. Cao, "Exponential stability of stochastic neural networks with both Markovian jump parameters and mixed time delays," IEEE Transactions on Systems, Man, and Cybernetics, Part B (Cybernetics), vol. 41, no. 2, pp. 341-353, 2011.

[32] F. Chen, Y. Yin, and F. Liu, "Delay-dependent robust fault detection for Markovian jump systems with partly unknown transition rates," Journal of the Franklin Institute, vol. 353, no. 2, pp. 426-447, 2016.

[33] Z.-G. Wu, J. H. Park, H. Su, and J. Chu, "Passivity analysis of Markov jump neural networks with mixed time-delays and piecewise-constant transition rates," Nonlinear Analysis: Real World Applications, vol. 13, no. 5, pp. 2423-2431, 2012.

[34] Y. Guo, "Improved synthesis method for Markov jump systems with uncertain transition rates," Journal of the Franklin Institute, vol. 352, no. 12, pp. 6011-6018, 2015.

[35] Y. Kao, J. Xie, and C. Wang, "Stabilization of singular Markovian jump systems with generally uncertain transition rates," IEEE Transactions on Automatic Control, vol. 59, no. 9, pp. 2604-2610, 2014.

[36] K. Babcock and R. Westervelt, "Stability and dynamics of simple electronic neural networks with added inertia," Physica D: Nonlinear Phenomena, vol. 23, no. 1-3, pp. 464-469, 1986.

[37] Z. Zhang and J. Cao, "Novel finite-time synchronization criteria for inertial neural networks with time delays via integral inequality method," IEEE Transactions on Neural Networks and Learning Systems, vol. 30, no. 5, pp. 1476-1485, 2019.

[38] S. Lu and Y. Gao, "Exponential stability in lagrange sense for inertial neural networks with time-varying delays," Neurocomputing, vol. 333, pp. 41-52, 2019.

[39] Z. Zhang, M. Chen, and A. Li, "Further study on finite-time synchronization for delayed inertial neural networks via inequality skills," Neurocomputing, vol. 373, pp. 15-23, 2020.

[40] C. Chen, L. Li, H. Peng, and Y. Yang, "Fixed-time synchronization of inertial memristor-based neural networks with discrete delay," Neural Networks, vol. 109, pp. 81-89, 2019.

[41] L. Wang, Z. Zeng, X. Zong, and M.-F. Ge, "Finite-time stabilization of memristor-based inertial neural networks with discontinuous activations and distributed delays," Journal of the Franklin Institute, vol. 356, no. 6, pp. 3628-3643, 2019.

[42] X. Zhong and Y. Gao, "Synchronization of inertial neural networks with time-varying delays via quantized sampled-data control," IEEE Transactions on Neural Networks and Learning Systems doi: 10.1109/TNNLS.2020.3026163, 2021.

[43] M. Prakash, P. Balasubramaniam, and S. Lakshmanan, "Synchronization of Markovian jumping inertial neural networks and its applications in image encryption," Neural Networks, vol. 83, pp. 86-93, 2016.

[44] S. Lakshmanan, M. Prakash, C. P. Lim, R. Rakkiyappan, P. Balasubramaniam, and S. Nahavandi, "Synchronization of an inertial neural network with time-varying delays and its application to secure communication," IEEE Transactions on Neural Networks and Learning Systems, vol. 29, no. 1, pp. 195-207, 2018.

[45] J. Wang, Z. Wang, X. Chen, and J. Qiu, "Synchronization criteria of delayed inertial neural networks with generally Markovian jumping," Neural Networks, vol. 139, pp. 64-76, 2021.

[46] Y. Kao, L. Shi, J. Xie, and H. R. Karimi, "Global exponential stability of delayed Markovian jump fuzzy cellular neural networks with generally incomplete transition probability," Neural Networks, vol. 63, pp. 18-30, 2015.

[47] Y. Liu, J. H. Park, B.-Z. Guo, F. Fang, and F. Zhou, "Event-triggered dissipative synchronization for Markovian jump neural networks with general transition probabilities," International Journal of Robust and Nonlinear Control, vol. 28, no. 13, pp. 3893-3908, 2018.

[48] Y. Liu, Z. Wang, and X. Liu, "Global exponential stability of generalized recurrent neural networks with discrete and distributed delays," Neural Networks, vol. 19, no. 5, pp. 667-675, 2006. 
[49] P. Park, J. W. Ko, and C. Jeong, "Reciprocally convex approach to stability of systems with time-varying delays," Automatica, vol. 47, no. 1, pp. 235-238, 2011.

[50] R. Datta, R. Dey, B. Bhattacharya, R. Saravanakumar, and O.-M. Kwon, "Stability and stabilization of T-S fuzzy systems with variable delays via new Bessel-Legendre polynomial based relaxed integral inequality," Information Sciences, vol. 522, pp. 99-123, 2020.

[51] P. Park, W. I. Lee, and S. Y. Lee, "Auxiliary function-based integral inequalities for quadratic functions and their applications to time-delay systems," Journal of the Franklin Institute, vol. 352, no. 4, pp. 1378$1396,2015$.

[52] J. Xiong and J. Lam, "Robust $H_{2}$ control of Markovian jump systems with uncertain switching probabilities," International Journal of Systems Science, vol. 40, no. 3, pp. 255-265, 2009.

[53] Z.-G. Wu, P. Shi, H. Su, and J. Chu, "Stochastic synchronization of Markovian jump neural networks with time-varying delay using sampled data," IEEE Transactions on Cybernetics, vol. 43, no. 6, pp. 1796-1806, 2013.

[54] C. Zhu, "A novel image encryption scheme based on improved hyperchaotic sequences," Optics Communications, vol. 285, no. 1, pp. 29-37, 2012.

[55] B. Norouzi, S. M. Seyedzadeh, S. Mirzakuchaki, and M. R. Mosavi, "A novel image encryption based on hash function with only two-round diffusion process," Multimedia Systems, vol. 20, no. 1, pp. 45-64, 2014. 